Als Manuskript gedruckt

Technische Universität Dresden

Herausgeber: Der Rektor

Local projection stabilisation on S-type meshes for convection-diffusion problems with characteristic layers

Sebastian Franz and Gunar Matthies

MATH-NM-07-2008

September 22, 2008 



\title{
Local projection stabilisation on S-type meshes for convection-diffusion problems with characteristic layers
}

\author{
Sebastian Franz and Gunar Matthies
}

September 22, 2008

\begin{abstract}
Singularly perturbed convection-diffusion problems with exponential and characteristic layers are considered on the unit square. The discretisation is based on layer-adapted meshes. The standard Galerkin method and the local projection scheme are analysed for bilinear and higher order finite element where enriched spaces were used. For bilinears, first order convergence in the $\varepsilon$-weighted energy norm is shown for both the Galerkin and the stabilised scheme. However, supercloseness results of second orders hold for the Galerkin method in the $\varepsilon$-weighted energy norm and for the local projection scheme in the corresponding norm. For the enriched $\mathcal{Q}_{p}$-elements, $p \geq 2$, which already contain the space $\mathcal{P}_{p+1}$, a convergence order $p+1$ in the $\varepsilon$-weighted energy norm is proved for both the Galerkin method and the local projection scheme. Furthermore, the local projection methods provides a supercloseness result of order $p+1$ in local projection norm.
\end{abstract}

MSC: 65N12, 65N30, 65N50

keywords: singular perturbation - characteristic layers - Shishkin meshes - local projection

\section{Introduction}

We consider the singularly perturbed model convection-diffusion equation

$$
\begin{aligned}
-\varepsilon \Delta u-b u_{x}+c u & =f & & \text { in } \Omega=(0,1)^{2}, \\
u & =0 & & \text { on } \Gamma=\partial \Omega,
\end{aligned}
$$

and shall assume that the data satisfy $b \in W_{\infty}^{1}(\Omega)$ and $c \in L_{\infty}(\Omega)$. Additionally, let $b \geq \beta$ on $\bar{\Omega}$ with some positive constant $\beta$, while $0<\varepsilon \ll 1$ is a small perturbation parameter. Its presence gives rise to an exponential layer of width $\mathcal{O}(\varepsilon)$ near the outflow boundary at $x=0$ and to two parabolic layers of width $\mathcal{O}(\sqrt{\varepsilon})$ near the characteristic boundaries at $y=0$ and $y=1$. A typical solution of (1.1) is shown in Fig. 1. 


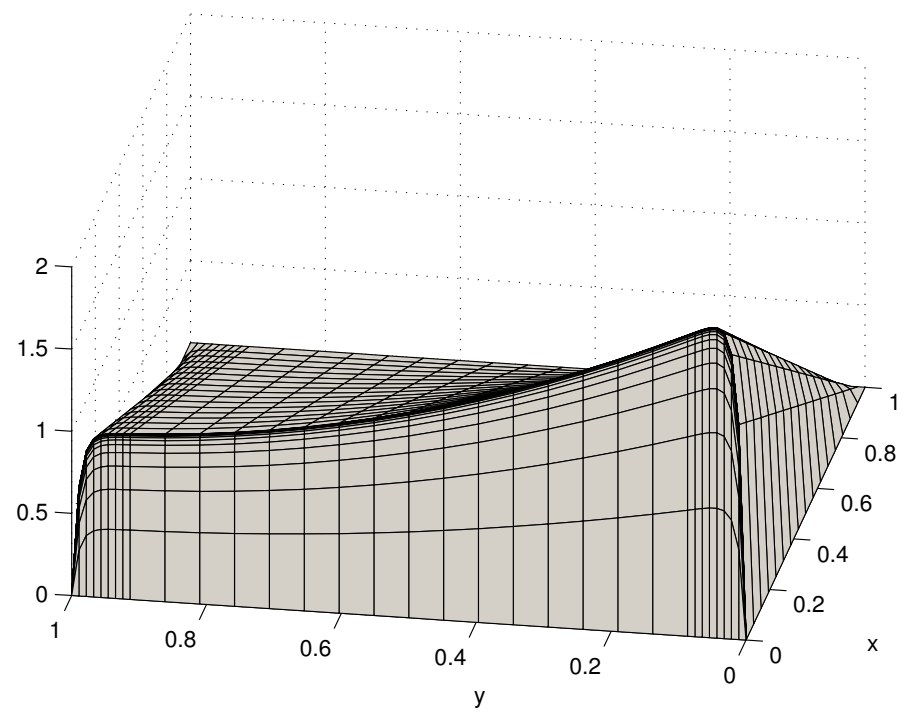

Figure 1: Typical solution of (1.1) with parabolic layers (left and right) and an exponential layer (front).

To ensure coercivity of the bilinear form associated with problem (1.1), we furthermore shall assume that

$$
c+\frac{1}{2} b_{x} \geq \gamma>0
$$

Therefore, problem (1.1) possesses a unique solution in $H_{0}^{1}(\Omega)$. Note that (1.2) can always be ensured by a simple transformation $\tilde{u}(x, y)=u(x, y) \mathrm{e}^{\varkappa x}$ with $\varkappa$ chosen suitably.

Due to the presence of layers, the use of quasi uniform meshes does not give accurate approximations of (1.1) unless the mesh size is of the order of the perturbation parameter $\varepsilon$ which in practice constitutes a prohibitive restriction. Therefore, layer-adapted meshes have to be used to obtain efficient discretisations. Based on a priori knowledge of the layer behaviour, we shall construct generalisations of Shishkin meshes, so called S-type meshes, that resolve the layers and yield robust (or uniform) convergence.

Bakhvalov [2] gave some early ideas on layer-adapted meshes. Piecewise uniform Shishkin meshes [25] were originally proposed for finite difference methods. Stynes and O'Riordan [27] were the first analysing the standard Galerkin finite element methods on Shishkin meshes. Linß $[19,20]$ considered the combination of Bakhvalov's idea for using a uniform coarse mesh and a graded fine mesh with Shishkin's choice of the transition point.

Due to the instability of the standard Galerkin method even on layer-adapted meshes, see [21] for numerical results, stabilised discretisations have to be considered.

The streamline-diffusion finite element method (SDFEM) was introduced by Hughes and Brooks [14]. The SDFEM gives good stability properties and highly accurate solutions outside the layers. For problems with characteristic layers, the SDFEM with bilinears was analysed in [13]. A disadvantage of the SDFEM which accounts in particular for 
discretisations with higher order elements is that several additional terms which include also second order derivatives have to be assembled in order to ensure the strong consistency of the resulting method.

One alternative stabilisation technique is the local projection method. The standard Galerkin finite element method is stabilised by adding terms which give a weighted control on the fluctuations $(i d-\pi)$ of certain or all derivatives of the quantity of interest where $\pi$ denoted a projection into a discontinuous finite element space. Originally proposed for the Stokes problem [3], the local projection method was extended to transport problems in [4]. The local projection stabilisation applied to the Oseen problem was analysed in [5,24]. In contrast to the SDFEM, the local projection method is only weakly consistent. However, the occurring consistency error can be bounded such that the optimal order of convergence is maintained.

The local projection method was originally proposed as a two level method where the projection space is defined on a coarser mesh consisting of patches of elements [3-5]. In this case, standard finite element spaces can be used for both the approximation space and the projection space. However, this approach shows a severe disadvantage since the discretisation stencil increases due to additional couplings introduced by the projection onto coarser meshes. Furthermore, additional data structures have to be provided within computer codes. The analysis of local projection methods is based on the existence of an interpolation operator which provides not only the standard interpolation error estimates but also an additional orthogonality property [24]. Using the abstract framework given in [24], the enrichment approach of the local projection method can be constructed where approximation space and projection space live on the same mesh. Compared to standard finite element spaces, the approximation space is enriched. It was shown in [24] that it suffices to enrich the standard $\mathcal{Q}_{p}$-element, $p \geq 2$, by just two additional bubble functions, independent of $p$. This ensures that the discretisation stencil remains small.

The local projection method on layer-adapted meshes for problems with exponential boundary layers was considered in $[22,23]$. In [23], new enriched finite elements were introduced. They are obtained by enriching the standard $\mathcal{Q}_{p}$-element, $p \geq 2$, by six functions resulting in an element which already contains the space $\mathcal{P}_{p+1}$. This provides better interpolation error estimates.

In contrary to singularly perturbed problems with exponential layers only, there is little known in literature about supercloseness analysis for problems with characteristic layers. These layers are different in structure and therefore the numerical analysis has to be adapted. For bilinear finite elements, a supercloseness analysis for problems like (1.1) was performed in [11-13] for the Galerkin method and several stabilisations like the SDFEM.

The main objective of this paper is the analysis of the local projection method for problems with characteristic layers on layer-adapted meshes. Both bilinear and higher order finite elements will be considered. For bilinear finite elements and the standard Galerkin method, we cite convergence and supercloseness results from [12]. If the local projection stabilisation is applied for bilinears, a second order supercloseness result between the bilinear interpolant of $u$ and the solution of stabilised discrete problem is obtained in the local projection norm. Furthermore, a first order convergence in the $\varepsilon$ weighted energy norm will be shown. For discretisations using the enriched $\mathcal{Q}_{p}$-elements, 
$p \geq 2$, which contain the space $\mathcal{P}_{p+1}$, a convergence order $p+1$ will be proved for the standard Galerkin method. Using the local projection stabilisation method, we will show a supercloseness result of order $p+1$ in the local projection norm and a convergence of order $p+1$ in the $\varepsilon$-weighted energy norm.

This paper is organised as follows. Section 2 describes the S-type meshes and their properties. The local projection stabilisation will be introduced in Section 3. The analysis of the local projection method applied discretisations with bilinear finite elements is considered in Subsection 3.1 while Subsection 3.2 handles the case of higher order finite elements. Numerical results for both bilinear and higher order elements will be given in Section 4.

Notation. Throughout this paper, $C$ denotes a generic constant that is independent of both the perturbation parameter $\varepsilon$ and the mesh parameter $N$. The dependence of any constant on the polynomial order $p$ will not be elaborated in this paper.

On an arbitrary measurable two-dimensional subset $D \subset \Omega$, the usual Sobolev spaces $W_{r}^{m}(D)$ and $L_{r}(D)$ are used. In the case $r=2$, we write $H^{m}(D)$ instead of $W_{2}^{m}(D)$. The $L_{2}(D)$-norm is denoted by $\|\cdot\|_{0, D}$ while the $L_{2}(D)$-inner product is written as $(\cdot, \cdot)_{D}$. Note that the subscript $D$ will be always dropped in the case $D=\Omega$.

\section{Solution decomposition and layer-adapted meshes}

As mentioned before, the solution $u$ of (1.1) has an exponential layer at $x=0$ and two parabolic layers at $y=0$ and $y=1$. We shall suppose for our later analysis that $u$ can be split into a regular solution component and various layer parts.

Assumption 1. The solution $u$ of (1.1) can be decomposed as

$$
u=v+w_{1}+w_{2}+w_{12}
$$

where we have for fixed $p \in \mathbb{N}$ and all $x, y \in[0,1]$ and $0 \leq i+j \leq p+2$ the pointwise estimates

$$
\left.\begin{array}{l}
\left|\frac{\partial^{i+j} v}{\partial x^{i} \partial y^{j}}(x, y)\right| \leq C, \quad\left|\frac{\partial^{i+j} w_{1}}{\partial x^{i} \partial y^{j}}(x, y)\right| \leq C \varepsilon^{-i} \mathrm{e}^{-\beta x / \varepsilon}, \\
\left|\frac{\partial^{i+j} w_{2}}{\partial x^{i} \partial y^{j}}(x, y)\right| \leq C \varepsilon^{-j / 2}\left(\mathrm{e}^{-y / \sqrt{\varepsilon}}+\mathrm{e}^{-(1-y) / \sqrt{\varepsilon}}\right), \\
\left|\frac{\partial^{i+j} w_{12}}{\partial x^{i} \partial y^{j}}(x, y)\right| \leq C \varepsilon^{-(i+j / 2)} \mathrm{e}^{-\beta x / \varepsilon}\left(\mathrm{e}^{-y / \sqrt{\varepsilon}}+\mathrm{e}^{-(1-y) / \sqrt{\varepsilon}}\right),
\end{array}\right\}
$$

where $w_{1}$ is the exponential boundary layer, $w_{2}$ the parabolic boundary layer, $w_{12}$ the corner layer, and $v$ the regular part.

Remark 2. In the bilinear case $p=1$ where actually pointwise bounds up to order 2 and only $L_{2}$-bounds up to order 3 are needed, see [12] for the analysis of the standard Galerkin method, Kellogg and Stynes [16, 17] proved the validity of the needed bounds for the case of constant functions $b, c$ provided $f \in C^{8, \alpha}(\bar{\Omega})$ satisfies the compatibility conditions

$$
f(0,0)=f(1,0)=f(1,1)=f(0,1)=0 .
$$


A similar analysis can be done here to obtain the required bounds on higher derivatives. This will result in additional smoothness and compatibility conditions on $f$.

When discretising (1.1), we use in both $x$ - and $y$-direction so called $S$-type meshes with $N$ mesh intervals in each direction which condense in the layer regions. We define for this purpose the mesh transition parameters

$$
\lambda_{x}:=\min \left\{\frac{1}{2}, \frac{\sigma \varepsilon}{\beta} \ln N\right\} \quad \text { and } \quad \lambda_{y}:=\min \left\{\frac{1}{4}, \sigma \sqrt{\varepsilon} \ln N\right\}
$$

with some user-chosen positive parameter $\sigma$ that will be fixed later.

The domain $\Omega$ is divided into four (six) subregions, see Fig. 2, with $\Omega_{12}$ covering the exponential layer, $\Omega_{21}$ the parabolic layer, $\Omega_{22}$ the corner layer and $\Omega_{11}$ the remaining non-layer region.

For the mere sake of simplicity in our subsequent analysis, we shall assume that

$$
\lambda_{x}=\frac{\sigma \varepsilon}{\beta} \ln N \leq \frac{1}{2} \quad \text { and } \quad \lambda_{y}=\sigma \sqrt{\varepsilon} \ln N \leq \frac{1}{4},
$$

as it is typically the case for (1.1).

Note that the mesh transition parameters $\lambda_{x}$ and $\lambda_{y}$ have been chosen such that the layer terms $w_{1}, w_{2}$, and $w_{12}$ of $u$ are of size $\mathcal{O}\left(N^{-\sigma}\right)$ on $\Omega_{11}$, i.e.,

$$
\left|w_{1}(x, y)\right|+\left|w_{2}(x, y)\right|+\left|w_{12}(x, y)\right| \leq C N^{-\sigma} \quad \text { for }(x, y) \in \Omega_{11} \text {. }
$$

The parameter $\sigma$ is typically chosen to equal the formal order of the method or to accommodate the error analysis.

The domain $\Omega$ will be dissected by a tensor product mesh according to

$$
\begin{aligned}
& x_{i}:= \begin{cases}\frac{\sigma \varepsilon}{\beta} \phi\left(\frac{i}{N}\right), & i=0, \ldots, N / 2, \\
1-2\left(1-\lambda_{x}\right)\left(1-\frac{i}{N}\right), & i=N / 2, \ldots, N,\end{cases} \\
& y_{j}:= \begin{cases}\sigma \sqrt{\varepsilon} \phi\left(\frac{2 j}{N}\right), & j=0, \ldots, N / 4, \\
\left(1-2 \lambda_{y}\right)\left(\frac{2 j}{N}-1\right)+\frac{1}{2}, & j=N / 4, \ldots, 3 N / 4, \\
1-\sigma \sqrt{\varepsilon} \phi\left(2-\frac{2 j}{N}\right), & j=3 N / 4, \ldots, N,\end{cases}
\end{aligned}
$$

\begin{tabular}{|l|l|}
\hline$\Omega_{22}$ & $\Omega_{21}$ \\
\hline$\Omega_{12}$ & $\Omega_{11}$ \\
& \\
\hline$\Omega_{22}$ & $\Omega_{21}$ \\
\hline
\end{tabular}

$$
\begin{aligned}
& \Omega_{11}:=\left[\lambda_{x}, 1\right] \times\left[\lambda_{y}, 1-\lambda_{y}\right], \\
& \Omega_{12}:=\left[0, \lambda_{x}\right] \times\left[\lambda_{y}, 1-\lambda_{y}\right], \\
& \Omega_{21}:=\left[\lambda_{x}, 1\right] \times\left(\left[0, \lambda_{y}\right] \cup\left[1-\lambda_{y}, 1\right]\right), \\
& \Omega_{22}:=\left[0, \lambda_{x}\right] \times\left(\left[0, \lambda_{y}\right] \cup\left[1-\lambda_{y}, 1\right]\right)
\end{aligned}
$$

Figure 2: Decomposition of $\Omega$ into subregions. 


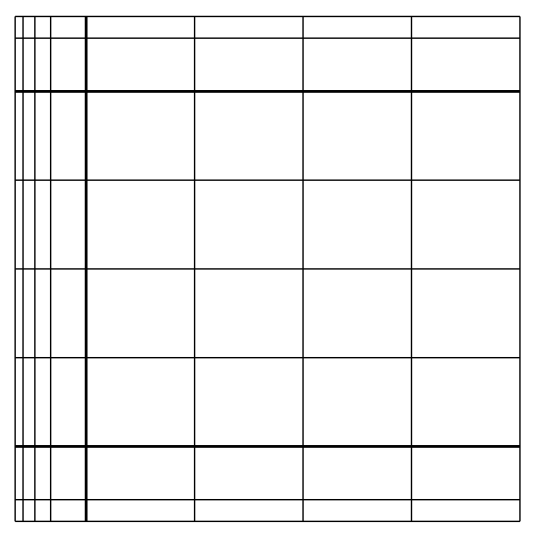

Figure 3: Triangulation $T^{8}$ of $\Omega$, the bold lines indicate the boundaries of the subdomains.

Table 1: Some examples of mesh-generating and mesh-characterising functions of S-type meshes.

\begin{tabular}{l|ll|ll} 
Name & $\phi(t)$ & $\max \phi^{\prime}$ & $\psi(t)$ & $\max \left|\psi^{\prime}\right|$ \\
\hline Shishkin mesh & $2 t \ln N$ & $2 \ln N$ & $N^{-2 t}$ & $2 \ln N$ \\
B-S mesh & $-\ln \left(1-2 t\left(1-N^{-1}\right)\right)$ & $2 N$ & $1-2 t\left(1-N^{-1}\right)$ & 2 \\
polynomial S-mesh & $(2 t)^{m} \ln N$ & $2 m \ln N$ & $N^{-(2 t)^{m}}$ & $C(\ln N)^{1 / m}$ \\
modified B-S mesh & $\frac{t}{q-t}, q=\frac{1}{2}\left(1+\frac{1}{\ln N}\right)$ & $3 \ln ^{2} N$ & $\mathrm{e}^{-\frac{t}{q-t}}$ & $3 /(2 q) \leq 3$
\end{tabular}

where $\phi$ is a monotonically increasing mesh-generating function satisfying $\phi(0)=0$ and $\phi(1 / 2)=\ln N$.

The final mesh is constructed by drawing lines parallel to the coordinate axes through these mesh points. The obtained triangulation is denoted by $T^{N}$. Fig. 3 shows an example of a triangulation. Note that $\Omega_{11}$ is dissected uniformly while the dissection in the other subdomains depends on $\phi$.

Related to the mesh-generating function $\phi$, we define by

$$
\psi=\mathrm{e}^{-\phi}
$$

the mesh-characterising function $\psi$ which is monotonically decreasing with $\psi(0)=1$ and $\psi(1 / 2)=N^{-1}$.

Tab. 1 gives some examples of S-type meshes using the naming convention introduced in [26]. The polynomial S-mesh has an additional parameter $m>0$ to adjust the grading inside the layer.

Later on, we need the following property.

Assumption 3. Let the mesh-generating function $\phi$ be piecewise differentiable such that

$$
\max _{t \in\left[0, \frac{1}{2}\right]} \phi^{\prime}(t) \leq C N \text { or equivalently } \max _{t \in\left[0, \frac{1}{2}\right]} \frac{\left|\psi^{\prime}(t)\right|}{\psi(t)} \leq C N
$$


is fulfilled.

Note that Assumption 3 is satisfied for all meshes given in Tab. 1.

Inside the fine mesh region, we can use (2.3) to estimate $h_{i}:=x_{i}-x_{i-1}$ from above. Let $t_{i}=i / N$. Then, it holds for $i=1, \ldots, N / 2\left(\right.$ with $\max \phi^{\prime}$ taken over $\left.t \in\left[t_{i-1}, t_{i}\right]\right)$

$$
\begin{aligned}
\psi\left(t_{i}\right)=\mathrm{e}^{-\phi\left(t_{i}\right)}=\mathrm{e}^{-\left(\phi\left(t_{i}\right)-\phi(t)\right)} \mathrm{e}^{-\phi(t)} & \geq \mathrm{e}^{-\left(\phi\left(t_{i}\right)-\phi\left(t_{i-1}\right)\right)} \psi(t) \\
& \geq \mathrm{e}^{-N^{-1} \max \phi^{\prime}} \psi(t) \geq C \psi(t) \quad \forall t \in\left[t_{i-1}, t_{i}\right] .
\end{aligned}
$$

Furthermore, we have

$$
x=\frac{\sigma \varepsilon}{\beta} \phi(t)=-\frac{\sigma \varepsilon}{\beta} \ln \psi(t), \quad \psi(t)=\mathrm{e}^{-\beta x /(\sigma \varepsilon)} .
$$

Using this, the monotonicity of $\psi$, and (2.4), we obtain for $i=1, \ldots, N / 2$ and $x \in\left[x_{i-1}, x_{i}\right]$

$$
\begin{aligned}
h_{i} & =\frac{\sigma \varepsilon}{\beta}\left(\phi\left(t_{i}\right)-\phi\left(t_{i-1}\right)\right) \leq \frac{\sigma}{\beta} \varepsilon N^{-1} \max _{t \in\left[t_{i-1}, t_{i}\right]} \phi^{\prime}(t) \leq \frac{\sigma}{\beta} \varepsilon N^{-1}\left(\max _{t \in\left[t_{i-1}, t_{i}\right]}\left|\psi^{\prime}(t)\right|\right) / \psi\left(t_{i}\right) \\
& \leq C \varepsilon N^{-1}\left(\max _{t \in\left[t_{i-1}, t_{i}\right]}\left|\psi^{\prime}(t)\right|\right) / \psi(t) \leq C \varepsilon N^{-1} \max \left|\psi^{\prime}\right| \mathrm{e}^{\beta x /(\sigma \varepsilon)}
\end{aligned}
$$

where $\max \left|\psi^{\prime}\right|:=\max _{t \in[0,1 / 2]}\left|\psi^{\prime}(t)\right|$.

Similarly, we get for $j=1, \ldots, N / 4$ and $j=3 N / 4+1, \ldots, N$

$$
k_{j}:=y_{j}-y_{j-1} \leq C \varepsilon^{1 / 2} N^{-1} \max \left|\psi^{\prime}\right| \begin{cases}\mathrm{e}^{y /\left(\sigma \varepsilon^{1 / 2}\right)}, & j \leq N / 4, \\ \mathrm{e}^{(1-y) /\left(\sigma \varepsilon^{1 / 2}\right)}, & j>3 N / 4,\end{cases}
$$

with $y \in\left[y_{j-1}, y_{j}\right]$. Of course, the simpler bounds

$$
\begin{array}{rlrl}
h_{i} \leq C \varepsilon N^{-1} \max \phi^{\prime} & \leq C \varepsilon, & i=1, \ldots, N / 2, \\
k_{j} \leq C \varepsilon^{1 / 2} N^{-1} \max \phi^{\prime} \leq C \varepsilon^{1 / 2}, & j=1, \ldots, N / 4,3 N / 4+1, \ldots, N,
\end{array}
$$

follow also from (2.3).

We need in the analysis for the higher order case the following property.

Assumption 4. The mesh generating function $\phi$ fulfils

$$
\min _{i=0, \ldots, N / 2-1}\left(\phi\left(\frac{i+1}{N}\right)-\phi\left(\frac{i}{N}\right)\right) \geq C N^{-1} .
$$

Remark 5. Assumption 4 allows to bound the mesh width in the layer regions from below. This is used while applying an inverse inequality in the layer regions, see the proofs of Theorems 13 and 15. Assumption 4 restricts the use of S-type meshes from Tab. 1. For the original Shishkin mesh, we have

$$
\min _{i=0, \ldots, N / 2-1}\left(\phi\left(\frac{i+1}{N}\right)-\phi\left(\frac{i}{N}\right)\right)=C N^{-1} \ln N \geq C N^{-1} .
$$


Both meshes of Bakhvalov-Shishkin type (B-S-mesh and modified B-S-mesh) fulfil

$$
\min _{i=0, \ldots, N / 2-1}\left(\phi\left(\frac{i+1}{N}\right)-\phi\left(\frac{i}{N}\right)\right) \geq C N^{-1} .
$$

Unfortunately, the polynomial S-type mesh yields

$$
\min _{i=0, \ldots, N / 2-1}\left(\phi\left(\frac{i+1}{N}\right)-\phi\left(\frac{i}{N}\right)\right) \geq C N^{-m}
$$

such that Assumption 4 fails for $m>1$.

Notation: Let

$$
h:=\max _{i=1, \ldots, N / 2} h_{i} \text { and } k:=\max _{j=1, \ldots, N / 4} k_{j}
$$

be the maximal mesh sizes inside the layer regions. We denote by $\tau_{i j}=\left[x_{i-1}, x_{i}\right] \times\left[y_{j-1}, y_{j}\right]$ a specific element and by $\tau$ a generic mesh rectangle. Note that the mesh cells are assumed to be closed.

\section{Local-projection stabilisation (LPS)}

The Galerkin bilinear form $a_{\text {Gal }}$ associated with problem (1.1) is defined by

$$
a_{G a l}(u, v):=\varepsilon(\nabla u, \nabla v)+\left(c u-b u_{x}, v\right), \quad u, v \in H_{0}^{1}(\Omega) .
$$

A weak formulation of the convection-diffusion problem (1.1) reads

Find $u \in H_{0}^{1}(\Omega)$ such that

$$
a_{\text {Gal }}(u, v)=(f, v) \quad \forall v \in H_{0}^{1}(\Omega) .
$$

Note that this problem is uniquely solvable due to (1.2).

Let $V^{N}$ be a conforming finite element space based on the triangulation $T^{N}$. The standard Galerkin formulation of (1.1) is given by

Find $\tilde{u}^{N} \in V^{N}$ such that

$$
a_{G a l}\left(\tilde{u}^{N}, v^{N}\right)=\left(f, v^{N}\right) \quad \forall v^{N} \in V^{N} .
$$

Due to (1.2), this problem possesses a unique solution. Furthermore, the Galerkin orthogonality

$$
a_{\text {Gal }}\left(u-\tilde{u}^{N}, v^{N}\right)=0 \quad \forall v^{N} \in V^{N}
$$

holds true.

Since the standard Galerkin discretisation lacks stability even on S-type meshes, see the numerical results given in [18], the local projection method is applied for stabilisation. To this end, we introduce some more notation. 
Let $\pi_{\tau}$ denote the $L_{2}$-projection into the finite dimensional function space $D(\tau)$ which will be given later. The fluctuation operator $\kappa_{\tau}: L_{2}(\tau) \rightarrow L_{2}(\tau)$ is defined by $\kappa_{\tau} v:=$ $v-\pi_{\tau} v$.

In order to get additional control on the derivative in streamline direction, we define the stabilisation term

$$
s(u, v):=\sum_{\tau \in T^{N}} \delta_{\tau}\left(\kappa_{\tau}\left(b u_{x}\right), \kappa_{\tau}\left(b v_{x}\right)\right)_{\tau}
$$

with the cell-dependent parameters $\delta_{\tau}, \tau \in T^{N}$, which be the specified later in the analysis. The parameter will be constant inside each subdomain of $\Omega$, i.e. $\delta_{\tau}=\left.\delta\right|_{\tau}=\delta_{i j}$ for $\tau \subset \Omega_{i j}$. It was stated in $[11,12]$ for different stabilisation methods that stabilisation is best if only applied in $\Omega_{11} \cup \Omega_{21}$. Therefore, we set $\delta_{12}=\delta_{22}=0$ in the following.

An alternative way for stabilisation would be to add the term

$$
g(u, v)=\sum_{\tau \in T^{N}} \delta_{\tau}\left(\kappa_{\tau}(\nabla u), \kappa_{\tau}(\nabla v)\right)_{\tau} .
$$

Using the stabilisation term $g$ within a local projection method for problems with characteristic layers would lead to a different scaling of the stabilisation parameter $\delta_{21}$ which would be proportional to $\varepsilon^{1 / 2}$ (using $g$ ) instead of $\varepsilon^{-1 / 2}$ (using $s$ ). The latter will be shown in Theorem 8 for bilinears and in Theorem 15 for higher order elements.

Due to the structure of $s$, the Cauchy-Schwarz-like estimate

$$
|s(v, w)| \leq(s(v, v))^{1 / 2}(s(w, w))^{1 / 2} \quad \forall v, w \in H_{0}^{1}(\Omega)
$$

holds true.

The stabilised bilinear form $a_{L P S}$ is defined by

$$
a_{L P S}(u, v):=a_{G a l}(u, v)+s(u, v), \quad u, v \in H_{0}^{1}(\Omega) .
$$

The stabilised discrete problem reads

Find $u^{N} \in V^{N}$ such that

$$
a_{L P S}\left(u^{N}, v^{N}\right)=\left(f, v^{N}\right) \quad \forall v^{N} \in V^{N} .
$$

The subsequent analysis uses the $\varepsilon$-weighted energy norm

$$
\|v \mid\|_{\varepsilon}:=\left(\varepsilon\|\nabla v\|_{0}^{2}+\gamma\|v\|_{0}^{2}\right)^{1 / 2}
$$

and the LPS-norm

$$
\|\mid v\|_{L P S}:=\left(\varepsilon\|\nabla v\|_{0}^{2}+\gamma\|v\|_{0}^{2}+s(v, v)\right)^{1 / 2} .
$$

The definition of the bilinear forms and norms gives immediately the coercivity

$$
a_{G a l}(v, v) \geq\|v\|_{\varepsilon}^{2}, \quad a_{L P S}(v, v) \geq\|v\|_{L P S}^{2}, \quad v \in H_{0}^{1}(\Omega) .
$$

Moreover, the solutions $u$ of (3.1) and $u^{N}$ of (3.5) fulfil the relation

$$
a_{L P S}\left(u-u^{N}, v^{N}\right)=s\left(u, v^{N}\right) \quad \forall v \in V^{N}
$$

due to the definition of the bilinear forms $a_{L P S}$ and $a_{G a l}$. 


\subsection{The bilinear case}

We start with the consideration of bilinear finite elements. To this end, let Assumption 1 be fulfilled with $p=1$. Actually, we only need weaker assumptions, namely pointwise estimates of Assumption 1 up to order 2, and $L_{2}$-bounds up to order 3, see Remark 2. Moreover, let

$$
V^{N}:=\left\{v \in C(\Omega):\left.v\right|_{\tau} \in \mathcal{Q}_{1}(\tau) \forall \tau \in T^{N}\right\}
$$

be the space of continuous, piecewise bilinear functions. We denote by $w^{I}$ the nodal interpolant of the function $w$. Furthermore, we choose $D(\tau)=\mathcal{P}_{0}(\tau)$ as projection space and $\sigma \geq 5 / 2$.

The anisotropic interpolation error bounds for the derivatives

$$
\begin{aligned}
& \left\|\left(w-w^{I}\right)_{x}\right\|_{L_{q}\left(\tau_{i j}\right)} \leq C\left\{h_{i}\left\|w_{x x}\right\|_{L_{q}\left(\tau_{i j}\right)}+k_{j}\left\|w_{x y}\right\|_{L_{q}\left(\tau_{i j}\right)}\right\}, \\
& \left\|\left(w-w^{I}\right)_{y}\right\|_{L_{q}\left(\tau_{i j}\right)} \leq C\left\{h_{i}\left\|w_{x y}\right\|_{L_{q}\left(\tau_{i j}\right)}+k_{j}\left\|w_{y y}\right\|_{L_{q}\left(\tau_{i j}\right)}\right\}
\end{aligned}
$$

were given in [1, Theorem 2.7] and hold true for $q \in[1, \infty]$ and arbitrary $w \in W_{q}^{2}\left(\tau_{i j}\right)$.

Similar estimates hold for the fluctuation operator $\kappa=\kappa_{\tau_{i j}}$,

$$
\begin{array}{ll}
\|\kappa w\|_{0, \tau_{i j}} \leq C\|w\|_{0, \tau_{i j}}, & w \in L_{2}\left(\tau_{i j}\right), \\
\|\kappa w\|_{0, \tau_{i j}} \leq C\left\{h_{i}\left\|w_{x}\right\|_{L_{p}\left(\tau_{i j}\right)}+k_{j}\left\|w_{y}\right\|_{L_{p}\left(\tau_{i j}\right)}\right\}, & w \in H^{1}\left(\tau_{i j}\right),
\end{array}
$$

see [6, Theorem 4.6.11].

Lemma 6 (Interpolation error). The interpolation error for the nodal bilinear interpolation can be estimated by

$$
\left\|u-u^{I}\right\|_{\varepsilon} \leq C\left(\varepsilon^{1 / 4} h+k+N^{-1} \max \left|\psi^{\prime}\right|\right)
$$

and the stabilisation term fulfils

$$
s\left(v-v^{I}, v-v^{I}\right) \leq C\left(\delta_{11} N^{-2}+\delta_{21} \varepsilon^{1 / 2} \ln N\left(k+N^{-1}\right)^{2}\right)
$$

where $v$ is the regular part of $u$ due to Assumption 1 .

Proof. The energy-norm estimate can be found in [12]. Using the stability (3.9a) and the anisotropic error estimate (3.8), we obtain

$$
\begin{aligned}
s\left(v-v^{I}, v-v^{I}\right)= & \sum_{\tau \subset \Omega_{11} \cup \Omega_{21}} \delta_{\tau}\left\|\kappa_{\tau}\left(b\left(v-v^{I}\right)_{x}\right)\right\|_{0, \tau}^{2} \\
\leq & C \sum_{\tau \subset \Omega_{11} \cup \Omega_{21}} \delta_{\tau}\left\|b\left(v-v^{I}\right)_{x}\right\|_{0, \tau}^{2} \\
\leq & C\left(\delta_{11} N^{-2}\left(\left\|v_{x x}\right\|_{0, \Omega_{11}}^{2}+\left\|v_{x y}\right\|_{0, \Omega_{11}}^{2}\right)+\right. \\
& \left.\quad \delta_{21}\left(N^{-2}\left\|v_{x x}\right\|_{0, \Omega_{21}}^{2}+k^{2}\left\|v_{x y}\right\|_{0, \Omega_{21}}^{2}\right)\right) \\
\leq & C\left(\delta_{11} N^{-2}+\delta_{21} \varepsilon^{1 / 2} \ln N\left(k+N^{-1}\right)^{2}\right)
\end{aligned}
$$

and the second assertion is proved. 
For the standard Galerkin bilinear form $a_{G a l}$, we cite from [12].

Lemma 7. The solution $\tilde{u}^{N}$ of the Galerkin problem (3.2) on an S-type mesh satisfies

$$
\left|a_{\text {Gal }}\left(u^{I}-u, u^{I}-\tilde{u}^{N}\right)\right| \leq C\left(\left(h+N^{-1}\right) \ln ^{1 / 4} N+k+N^{-1} \max \left|\psi^{\prime}\right|\right)^{2}\left|\left\|\tilde{u}^{N}-u^{I} \mid\right\|_{\varepsilon}\right.
$$

where $u$ is the solution of (3.1) and $u^{I}$ its bilinear interpolant. Moreover, we have

$$
\left\|u^{N}-u^{I}\right\|_{\varepsilon} \leq C\left(\left(h+N^{-1}\right) \ln ^{1 / 4} N+k+N^{-1} \max \left|\psi^{\prime}\right|\right)^{2} .
$$

and error estimate

$$
\left\||| u-\tilde{u}^{N}\left|\left\|_{\varepsilon} \leq\right\|\right| u-u^{I}\left|\left\|_{\varepsilon}+\right\|\right|\left|u^{I}-\tilde{u}^{N}\right|\right\|_{\varepsilon} \leq C\left(\varepsilon^{1 / 4} h+k+N^{-1} \max \left|\psi^{\prime}\right|\right) .
$$

holds true.

Theorem 8 (Supercloseness and Convergence LPS-FEM). Let the stabilisation parameter be chosen according to

$$
\delta_{11} \leq C N^{-2}\left(\max \left|\psi^{\prime}\right|\right)^{4}, \quad \delta_{21} \leq C \varepsilon^{-1 / 2} \ln ^{-1} N\left(k+N^{-1} \max \left|\psi^{\prime}\right|\right)^{2}, \quad \delta_{12}=\delta_{22}=0 .
$$

Then, the numerical solution $u^{N}$ of (3.5) satisfies

$$
\left\|u^{I}-u^{N}\right\|_{L P S} \leq C\left(\left(h+N^{-1}\right) \ln ^{1 / 4} N+k+N^{-1} \max \left|\psi^{\prime}\right|\right)^{2}
$$

and

$$
\left\|\mid u-u^{N}\right\|_{\varepsilon} \leq C\left(\varepsilon^{1 / 4} h+k+N^{-1} \max \left|\psi^{\prime}\right|\right)
$$

where $u$ is the solution of (3.1) and $u^{I}$ its bilinear interpolant.

Proof. We start with coercivity and weak orthogonality to get

$$
\left\|\mid u^{I}-u^{N}\right\|_{L P S}^{2} \leq a_{L P S}\left(u^{I}-u^{N}, u^{I}-u^{N}\right)=a_{G a l}\left(u^{I}-u, u^{I}-u^{N}\right)+s\left(u^{I}, u^{I}-u^{N}\right) .
$$

The first term is bounded by (3.10). We use for the second term the decomposition $u=v+w_{1}+w_{2}+w_{12}$ according to Assumption 1 and expand the stabilisation term with $v$ as

$$
s\left(v^{I}, w^{N}\right)=s\left(v^{I}-v, w^{N}\right)+s\left(v, w^{N}\right) .
$$

Applying (3.4) for an arbitrary function $w$, we get

$$
\left|s\left(w, w^{N}\right)\right| \leq s(w, w)^{1 / 2} s\left(w^{N}, w^{N}\right)^{1 / 2} \leq\left(\sum_{\tau \subset \Omega_{11} \cup \Omega_{21}} \delta_{\tau}\left\|\kappa_{\tau}\left(b w_{x}\right)\right\|_{0, \tau}^{2}\right)^{1 / 2}\left\|w^{N}\right\| \|_{L P S} .
$$


Involving this, the first term in (3.13) can be bounded by using the estimate provided by Lemma 6. To get the bound for the second term in (3.13), we have to estimate above sum for $w=v$. Applying (3.9b), we get

$$
\begin{aligned}
\sum_{\tau \subset \Omega_{11} \cup \Omega_{21}} \delta_{\tau}\left\|\kappa_{\tau}\left(b v_{x}\right)\right\|_{0, \tau}^{2} \leq & C\left(\delta_{11} N^{-2}\left(\left\|\left(b v_{x}\right)_{x}\right\|_{0, \Omega_{11}}^{2}+\left\|\left(b v_{x}\right)_{y}\right\|_{0, \Omega_{11}}^{2}\right)+\right. \\
& \left.\delta_{21}\left(N^{-2}\left\|\left(b v_{x}\right)_{x}\right\|_{0, \Omega_{21}}^{2}+k^{2}\left\|\left(b v_{x}\right)_{y}\right\|_{0, \Omega_{21}}^{2}\right)\right) \\
\leq & C\left(\delta_{11} N^{-2}+\delta_{21} \varepsilon^{1 / 2} \ln N\left(k+N^{-1}\right)^{2}\right)
\end{aligned}
$$

Thus, we have

$$
\left|s\left(v^{I}, w^{N}\right)\right| \leq\left(\delta_{11} N^{-2}+\delta_{21} \varepsilon^{1 / 2} \ln N\left(k+N^{-1}\right)^{2}\right)^{1 / 2} \|\left.\left|w^{N}\right|\right|_{L P S} .
$$

Now let us turn to the layer parts $w=w_{1}+w_{12}$. These are small on $\Omega_{11} \cup \Omega_{21}$ due to their exponential decay. Thus, we estimate directly

$$
\begin{aligned}
\sum_{\tau \subset \Omega_{11} \cup \Omega_{21}} \delta_{\tau}\left\|\kappa_{\tau}\left(b w_{x}^{I}\right)\right\|_{0, \tau}^{2} & \leq C \sum_{\tau \subset \Omega_{11} \cup \Omega_{21}} \delta_{\tau}\left\|b w_{x}^{I}\right\|_{0, \tau}^{2} \\
& \leq C\left(\delta_{11}\left\|w_{x}^{I}\right\|_{0, \Omega_{11}}^{2}+\delta_{21}\left\|w_{x}^{I}\right\|_{0, \Omega_{21}}^{2}\right) \\
& \leq C N^{2}\left(\delta_{11}\left\|w^{I}\right\|_{0, \Omega_{11}}^{2}+\delta_{21}\left\|w^{I}\right\|_{0, \Omega_{21}}^{2}\right) \\
& \leq C N^{2}\left(\delta_{11}\left\|w^{I}\right\|_{L_{\infty}\left(\Omega_{11}\right)}^{2}+\delta_{21} \varepsilon^{1 / 2} \ln N\left\|w^{I}\right\|_{L_{\infty}\left(\Omega_{21}\right)}^{2}\right) \\
& \leq C N^{2}\left(\delta_{11}\|w\|_{L_{\infty}\left(\Omega_{11}\right)}^{2}+\delta_{21} \varepsilon^{1 / 2} \ln N\|w\|_{L_{\infty}\left(\Omega_{21}\right)}^{2}\right) \\
& \leq C\left(\delta_{11}+\delta_{21} \varepsilon^{1 / 2} \ln N\right) N^{-2(\sigma-1)}
\end{aligned}
$$

using an inverse estimate and the stability of the nodal interpolation with respect to the $L_{\infty}$-norm. It follows

$$
\left|s\left(\left(w_{1}+w_{12}\right)^{I}, w^{N}\right)\right| \leq\left. C\left(\delta_{11}+\delta_{21} \varepsilon^{1 / 2} \ln N\right)^{1 / 2} N^{-\sigma+1}||\left|w^{N}\right|\right|_{L P S} .
$$

To bound the remaining part $w_{2}$, we combine the techniques used above. On $\Omega_{11}$ the decay of $w_{2}$ is used while on $\Omega_{21}$ the interpolation error and (2.6) are exploited. We obtain

$$
\begin{aligned}
\left|s\left(w_{2}^{I}, w^{N}\right)_{\Omega_{11}}\right| & \leq\left. C \delta_{11}^{1 / 2} N^{-\sigma+1}||\left|w^{N}\right|\right|_{L P S} \\
\left|s\left(w_{2}^{I}, w^{N}\right)_{\Omega_{21}}\right| & \leq\left|s\left(w_{2}^{I}-w_{2}, w^{N}\right)_{\Omega_{21}}\right|+\left|s\left(w_{2}, w^{N}\right)_{\Omega_{21}}\right| \\
& \leq C\left(\delta_{21} \varepsilon^{1 / 2}\right)^{1 / 2} N^{-1} \max \left|\psi^{\prime}\right| .
\end{aligned}
$$

Using the bounds

$$
\delta_{11} \leq C N^{-2}\left(\max \left|\psi^{\prime}\right|\right)^{4}, \quad \delta_{21} \leq C \varepsilon^{-1 / 2} \ln ^{-1} N\left(k+N^{-1} \max \left|\psi^{\prime}\right|\right)^{2}
$$

and the estimates (3.14)-(3.16) together with (3.10) completes the proof. Please note that $\varepsilon^{-1 / 2} \ln ^{-1} N \geq 4 \sigma \geq 10$ holds true due to (2.2). Estimate (3.12) follows directly from (3.11) and Lemma 6. 
Remark 9. The quality of the numerical solution can be enhanced with a simple postprocessing routine to

$$
\left\|u-P u^{N}\right\|_{\varepsilon} \leq C\left(\left(h+N^{-1}\right) \ln ^{1 / 4} N+k+N^{-1} \max \left|\psi^{\prime}\right|\right)^{2},
$$

see [12, 28].

\subsection{The higher order case}

We turn now to higher order discretisations of the convection-diffusion problem (1.1). Let Assumption 1 be fulfilled for a fixed integer $p \geq 2, \sigma \geq p+2$ and Assumption 4 be true. Since we are not aware of supercloseness results for the standard Galerkin discretisation with $\mathcal{Q}_{p}$-elements on S-type meshes, we will use enriched spaces which were introduced in [23]. To this end, we recall their definition.

Let $\hat{\tau}=[-1,1]^{2}$ denote the reference element. We set

$$
\begin{aligned}
& \widehat{\mathcal{Q}}_{p}^{+}(\hat{\tau}):=\widehat{\mathcal{Q}}_{p}(\hat{\tau}) \oplus \operatorname{span}\{\left.\left(1-\xi^{2}\right)\left(1-\eta^{2}\right) \xi^{p-1},\left(1-\xi^{2}\right)\left(1-\eta^{2}\right) \eta^{p-1}\right\} \\
& \oplus \operatorname{span}\left\{(1+\xi)\left(1-\eta^{2}\right) L_{p-1}(\eta),(1-\xi)\left(1-\eta^{2}\right) L_{p-1}(\eta),\right. \\
&\left.(1+\eta)\left(1-\xi^{2}\right) L_{p-1}(\xi),(1-\eta)\left(1-\xi^{2}\right) L_{p-1}(\xi)\right\}
\end{aligned}
$$

with $L_{i}(\xi)$ denoting the one-dimensional Legendre polynomial of degree $i>0$, normalised to $L_{i}(1)=1$. Using the reference mapping $F_{\tau}: \hat{\tau} \rightarrow \tau$, we get $\mathcal{Q}_{p}^{+}(\tau)$. Our discrete space is now given by

$$
V^{N}:=\left\{v \in C(\Omega):\left.v\right|_{\tau} \in \mathcal{Q}_{p}^{+}(\tau) \forall \tau \in T^{N}\right\}
$$

Let $a_{i}$ and $e_{i}, i=1, \ldots, 4$, denote the vertices and edges of $\tau$, respectively. We define the interpolation operator $I_{\tau}: C(\tau) \rightarrow \mathcal{Q}_{p}^{+}(\tau)$ by

$$
\begin{array}{rlrl}
I_{\tau} v\left(a_{i}\right) & =v\left(a_{i}\right), \quad i=1, \ldots, 4 & & \\
\int_{e_{i}}\left(I_{\tau} v\right) q & =\int_{e_{i}} v q, \quad i=1, \ldots, 4, \quad q \in \mathcal{P}_{p-1}\left(e_{i}\right) \\
\int_{\tau}\left(I_{\tau} v\right) q & =\int_{\tau} v q, & & q \in \mathcal{Q}_{p-2}(\tau) \cup \mathcal{P}_{p-1}(\tau) .
\end{array}
$$

Due to [23, Lemma 3], this interpolation operator is uniquely defined and can be extended to the global interpolation operator $I^{N}: C(\bar{\Omega}) \rightarrow V^{N}$ in the usual way by

$$
\left.\left(I^{N} v\right)\right|_{\tau}:=I_{\tau}\left(\left.v\right|_{\tau}\right) \quad \forall \tau \in T^{N}, v \in C(\bar{\Omega}) .
$$

Lemma 10. The interpolation operator $I^{N}: C(\bar{\Omega}) \rightarrow V^{N}$ defined by (3.17) fulfils the orthogonality property

$$
\left(w-I^{N} w, q\right)_{\tau}=0 \quad \forall \tau \in T^{N}, q \in \mathcal{P}_{p-1}(\tau), w \in C(\tau)
$$


and the stability property

$$
\left\|I^{N} w\right\|_{L_{\infty}(\tau)} \leq C\|w\|_{L_{\infty}(\tau)} \quad \forall w \in C(\tau) .
$$

Moreover, the anisotropic error estimates

$$
\begin{gathered}
\left\|w-I^{N} w\right\|_{L_{q}\left(\tau_{i j}\right)} \leq C \sum_{r=0}^{s}\left\|h_{i}^{s-r} k_{j}^{r} \frac{\partial^{s} w}{\partial x^{s-r} \partial y^{r}}\right\|_{L_{q}\left(\tau_{i j}\right)}, \\
\left\|\left(w-I^{N} w\right)_{x}\right\|_{L_{q}\left(\tau_{i j}\right)} \leq C \sum_{r=0}^{t}\left\|h_{i}^{t-r} k_{j}^{r} \frac{\partial^{t+1} w}{\partial x^{t-r+1} \partial y^{r}}\right\|_{L_{q}\left(\tau_{i j}\right)}, \\
\left\|\left(w-I^{N} w\right)_{y}\right\|_{L_{q}\left(\tau_{i j}\right)} \leq C \sum_{r=0}^{t}\left\|h_{i}^{t-r} k_{j}^{r} \frac{\partial^{t+1} w}{\partial x^{t-r} \partial y^{r+1}}\right\|_{L_{q}\left(\tau_{i j}\right)}
\end{gathered}
$$

hold true for $q \in[1, \infty], 2 \leq s \leq p+2$, and $1 \leq t \leq p+1$.

Proof. The orthogonality (3.18) is a direct consequence of (3.17c). For the proof of (3.19), see [23, Lemma 5], The anisotropic error estimates follow from [23, Lemmas 6-7].

For the discretisation with the enriched space $\mathcal{Q}_{p}^{+}$, we choose $D(\tau)=\mathcal{P}_{p-1}(\tau)$ as projection space. The following estimate is a direct consequence of the Bramble-Hilbert lemma.

Lemma 11. Let $0 \leq s \leq p$ and $w \in H^{s}\left(\tau_{i j}\right)$. Then, the estimate

$$
\left\|\kappa_{\tau_{i j}}(w)\right\|_{0, \tau_{i j}}=\left\|w-\pi_{\tau_{i j}}(w)\right\|_{0, \tau_{i j}} \leq C \sum_{r=0}^{s}\left\|h_{i}^{s-r} k_{j}^{r} \frac{\partial^{s} w}{\partial x^{s-r} \partial y^{r}}\right\|_{0, \tau_{i j}}
$$

holds true.

Theorem 12 (Interpolation error). Let $u$ denote the solution of (3.1). Then, the interpolation operator $I^{N}$ defined above provides the following pointwise interpolation error bounds

$$
\begin{aligned}
& \left\|I^{N} u-u\right\|_{L_{\infty}\left(\Omega_{11}\right)} \leq C N^{-(p+2)}, \\
& \left\|I^{N} u-u\right\|_{L_{\infty}\left(\Omega_{12}\right)} \leq C\left(h+N^{-1} \max \left|\psi^{\prime}\right|\right)^{p+2}, \\
& \left\|I^{N} u-u\right\|_{L_{\infty}\left(\Omega_{21}\right)} \leq C\left(k+N^{-1} \max \left|\psi^{\prime}\right|\right)^{p+2}, \\
& \left\|I^{N} u-u\right\|_{L_{\infty}\left(\Omega_{22}\right)} \leq C\left(h+k+N^{-1} \max \left|\psi^{\prime}\right|\right)^{p+2} .
\end{aligned}
$$

Moreover, the $L_{2}$-estimates

$$
\begin{aligned}
\left\|I^{N} u-u\right\|_{0} & \leq C\left(k+h+N^{-1} \max \left|\psi^{\prime}\right|\right)^{p+2}, \\
\left\|I^{N} u-u\right\|_{0, \Omega_{11} \cup \Omega_{21}} & \leq C\left(k+N^{-1} \max \left|\psi^{\prime}\right|\right)^{p+2},
\end{aligned}
$$


and energy norm bound

$$
\left\|\mid I^{N} u-u\right\|_{\varepsilon} \leq C\left(k+h+N^{-1} \max \left|\psi^{\prime}\right|\right)^{p+1}
$$

hold true. Furthermore, it holds

$$
\left|s\left(I^{N} v-v, I^{N} v-v\right)\right| \leq C\left(\delta_{11} N^{-2(p+1)}+\delta_{21} \varepsilon^{1 / 2} \ln N\left(k+N^{-1}\right)^{2(p+1)}\right)
$$

where $v$ is the regular part of $u$ due to Assumption 1.

Proof. We start with the pointwise bounds where we will use the decomposition $u=$ $v+w_{1}+w_{2}+w_{12}$ due to Assumption 1. The local estimate (3.20a) with $q=\infty$ and $s=p+2$ yields

$$
\begin{aligned}
\left\|v-I^{N} v\right\|_{L_{\infty}\left(\Omega_{11}\right)} & \leq C \max _{\tau_{i j} \subset \Omega_{11}}\left\{\sum_{r=0}^{p+2} h_{i}^{p+2-r} k_{j}^{r}\left\|\frac{\partial^{p+2} v}{\partial x^{p+2-r} \partial y^{r}}\right\|_{L_{\infty}\left(\tau_{i j}\right)}\right\} \\
& \leq C N^{-(p+2)} \\
\left\|v-I^{N} v\right\|_{L_{\infty}\left(\Omega_{12}\right)} & \leq C\left(h+N^{-1}\right)^{p+2}, \\
\left\|v-I^{N} v\right\|_{L_{\infty}\left(\Omega_{21}\right)} & \leq C\left(k+N^{-1}\right)^{p+2}, \\
\left\|v-I^{N} v\right\|_{L_{\infty}\left(\Omega_{22}\right)} & \leq C(h+k)^{p+2} .
\end{aligned}
$$

The $L_{\infty}$-stability of the interpolation operator gives on $\Omega_{11}$ for the layer terms $w=$ $w_{1}+w_{2}+w_{12}$ the estimate

$$
\left\|w-I^{N} w\right\|_{L_{\infty}\left(\Omega_{11}\right)} \leq\|w\|_{L_{\infty}\left(\Omega_{11}\right)}+\left\|I^{N} w\right\|_{L_{\infty}\left(\Omega_{11}\right)} \leq C\|w\|_{L_{\infty}\left(\Omega_{11}\right)} \leq C N^{-\sigma} .
$$

We obtain similarly

$$
\begin{aligned}
& \left\|\left(w_{1}+w_{12}\right)-I^{N}\left(w_{1}+w_{12}\right)\right\|_{L_{\infty}\left(\Omega_{21}\right)} \leq C N^{-\sigma} \\
& \left\|\left(w_{2}+w_{12}\right)-I^{N}\left(w_{2}+w_{12}\right)\right\|_{L_{\infty}\left(\Omega_{12}\right)} \leq C N^{-\sigma}
\end{aligned}
$$

Using (3.20a) and (2.5) on $\Omega_{12} \cup \Omega_{22}$ results in

$$
\begin{aligned}
\left\|w_{1}-I^{N} w_{1}\right\|_{L_{\infty}\left(\Omega_{12} \cup \Omega_{22}\right)} & \leq C \max _{\tau_{i j} \subset \Omega_{12} \cup \Omega_{22}}\left\{\sum_{r=0}^{p+2}\left\|h_{i}^{p+2-r} k_{j}^{r} \frac{\partial^{p+2} w_{1}}{\partial x^{p+2-r} \partial y^{r}}\right\|_{L_{\infty}\left(\tau_{i j}\right)}\right\} \\
& \leq C \max _{\tau_{i j}}\left\{\sum_{r=0}^{p+2}\left(\varepsilon N^{-1} \max \left|\psi^{\prime}\right|\right)^{p+2-r}\left(k+N^{-1}\right)^{r} \times\right. \\
& \leq C\left(k+N^{-1} \max \left|\psi^{\prime}\right|\right)^{p+2} .
\end{aligned}
$$


The same technique provides for $w_{2}$ on $\Omega_{21} \cup \Omega_{22}$

$$
\left\|w_{2}-I^{N} w_{2}\right\|_{L_{\infty}\left(\Omega_{21} \cup \Omega_{22}\right)} \leq C\left(h+N^{-1} \max \left|\psi^{\prime}\right|\right)^{p+2}
$$

and for $w_{12}$ on $\Omega_{22}$

$$
\left\|w_{12}-I^{N} w_{12}\right\|_{L_{\infty}\left(\Omega_{22}\right)} \leq C\left(N^{-1} \max \left|\psi^{\prime}\right|\right)^{p+2} .
$$

Combining (3.27)-(3.29) proves the pointwise bounds (3.22).

The $L_{2}$-bounds (3.23) and (3.24) are immediate consequences of the just proved pointwise bounds.

To estimate the interpolation error in the $H^{1}$-seminorm as needed for the energy norm estimate (3.25), basically three techniques are used. The first one uses the local estimates (3.20b) and (3.20c) with $q=2$ and $t=p+1$. For an arbitrary $w$ on a subdomain $\Omega^{*} \subset \Omega$, it follows

$$
\begin{aligned}
& \left\|\left(w-I^{N} w\right)_{x}\right\|_{0, \Omega^{*}}^{2} \leq C \sum_{\tau_{i j} \subset \Omega^{*}} \sum_{r=0}^{p+1}\left\|h_{i}^{p+1-r} k_{j}^{r} \frac{\partial^{p+2} w}{\partial x^{p+2-r} \partial y^{r}}\right\|_{0, \tau_{i j}}^{2}, \\
& \left\|\left(w-I^{N} w\right)_{y}\right\|_{0, \Omega^{*}}^{2} \leq C \sum_{\tau_{i j} \subset \Omega^{*}} \sum_{r=0}^{p+1}\left\|h_{i}^{p+1-r} k_{j}^{r} \frac{\partial^{p+2} w}{\partial x^{p+1-r} \partial y^{r+1}}\right\|_{0, \tau_{i j}}^{2} .
\end{aligned}
$$

The second technique uses (3.20b) and (3.20c) with $q=2$ and $t=1$. It gives

$$
\begin{aligned}
& \left\|\left(w-I^{N} w\right)_{x}\right\|_{0, \Omega^{*}}^{2} \leq C \sum_{\tau_{i j} \subset \Omega^{*}}\left(\left\|h_{i} w_{x x}\right\|_{0, \tau_{i j}}^{2}+\left\|k_{j} w_{x y}\right\|_{0, \tau_{i j}}^{2}\right), \\
& \left\|\left(w-I^{N} w\right)_{y}\right\|_{0, \Omega^{*}}^{2} \leq C \sum_{\tau_{i j} \subset \Omega^{*}}\left(\left\|h_{i} w_{x y}\right\|_{0, \tau_{i j}}^{2}+\left\|k_{j} w_{y y}\right\|_{0, \tau_{i j}}^{2}\right) .
\end{aligned}
$$

A third method is applied to those terms which are already decayed. We use an inverse estimate and the $L_{\infty}$-stability of the interpolation operator. Denoting the minimal mesh size in $x$-direction inside $\Omega^{*}$ by $h_{\min , \Omega^{*}}$, we obtain

$$
\begin{aligned}
\left\|\left(w-I^{N} w\right)_{x}\right\|_{0, \Omega^{*}} & \leq\left\|w_{x}\right\|_{0, \Omega^{*}}+\left\|\left(I^{N} w\right)_{x}\right\|_{0, \Omega^{*}} \\
& \leq C\left(\left\|w_{x}\right\|_{0, \Omega^{*}}+h_{\min , \Omega^{*}}^{-1} \operatorname{meas}^{1 / 2}\left(\Omega^{*}\right)\|w\|_{L_{\infty}\left(\Omega^{*}\right)}\right) .
\end{aligned}
$$

We start with estimating the non-layer term $v$. Applying (3.30) to both derivatives yields with the pointwise bounds of Assumption 1

$$
\begin{aligned}
& \left\|\nabla\left(v-I^{N} v\right)\right\|_{0, \Omega_{11}} \leq C N^{-(p+1)}, \\
& \left\|\nabla\left(v-I^{N} v\right)\right\|_{0, \Omega_{12}} \leq C \varepsilon^{1 / 2} \ln ^{1 / 2} N\left(h+N^{-1}\right)^{p+1}, \\
& \left\|\nabla\left(v-I^{N} v\right)\right\|_{0, \Omega_{21}} \leq C \varepsilon^{1 / 4} \ln ^{1 / 2} N\left(k+N^{-1}\right)^{p+1}, \\
& \left\|\nabla\left(v-I^{N} v\right)\right\|_{0, \Omega_{22}} \leq C \varepsilon^{3 / 4} \ln N(h+k)^{p+1} .
\end{aligned}
$$


We have $h_{\min , \Omega_{11}} \geq C N^{-1}$. Thus, (3.32) gives for $w=w_{1}+w_{2}+w_{12}$

$$
\left\|\nabla\left(w-I^{N} w\right)\right\|_{0, \Omega_{11}} \leq C\left(\varepsilon^{-1 / 2} N^{-\sigma}+N\|w\|_{L_{\infty}\left(\Omega_{11}\right)}\right) \leq C \varepsilon^{-1 / 2} N^{-(p+1)}
$$

Taking $h_{\min , \Omega_{21}} \geq C N^{-1}$ into consideration, we obtain for the $x$-derivative of $w_{1}$ on $\Omega_{21}$ the estimate

$$
\left\|\left(w_{1}-I^{N} w_{1}\right)_{x}\right\|_{0, \Omega_{21}} \leq C \varepsilon^{-1 / 2} N^{-(p+1)} .
$$

Using (3.31), we get for the $y$-derivative of $w_{1}$ on $\Omega_{21}$

$$
\left\|\left(w_{1}-I^{N} w_{1}\right)_{y}\right\|_{0, \Omega_{21}} \leq C\left(N^{-1}\left\|w_{1 x y}\right\|_{0, \Omega_{21}}+k\left\|w_{1 y y}\right\|_{0, \Omega_{21}}\right) \leq C \varepsilon^{-1 / 4} N^{-(p+2)} .
$$

We obtain for the $x$-derivative of $w_{1}$ on $\Omega_{12} \cup \Omega_{22}$ with (3.30) and (2.5)

$$
\begin{aligned}
\left\|\left(w_{1}-I^{N} w_{1}\right)_{x}\right\|_{0, \Omega_{12} \cup \Omega_{22}} & \leq C \sum_{r=0}^{p+1}\left(\varepsilon N^{-1} \max \left|\psi^{\prime}\right|\right)^{p+1-r}\left(k+N^{-1}\right)^{r} \times \\
& \left\|\mathrm{e}^{(p+1-r) \beta x /(\sigma \varepsilon)} \frac{\partial^{p+2} w_{1}}{\partial x^{p+2-r} \partial y^{r}}\right\|_{0, \Omega_{12} \cup \Omega_{22}} \\
& \leq C \varepsilon^{-1 / 2}\left(k+N^{-1} \max \left|\psi^{\prime}\right|\right)^{p+1}
\end{aligned}
$$

and similarly

$$
\begin{aligned}
\left\|\left(w_{1}-I^{N} w_{1}\right)_{y}\right\|_{0, \Omega_{12} \cup \Omega_{22}} & \leq C \varepsilon^{1 / 2}\left(k+N^{-1} \max \left|\psi^{\prime}\right|\right)^{p+1}, \\
\left\|\left(w_{2}-I^{N} w_{2}\right)_{x}\right\|_{0, \Omega_{21} \cup \Omega_{22}} & \leq C \varepsilon^{1 / 4}\left(h+N^{-1} \max \left|\psi^{\prime}\right|\right)^{p+1}, \\
\left\|\left(w_{2}-I^{N} w_{2}\right)_{y}\right\|_{0, \Omega_{21} \cup \Omega_{22}} & \leq C \varepsilon^{-1 / 4}\left(h+N^{-1} \max \left|\psi^{\prime}\right|\right)^{p+1} \\
\left\|\left(w_{12}-I^{N} w_{12}\right)_{x}\right\|_{0, \Omega_{22}} & \leq C \varepsilon^{-1 / 4}\left(N^{-1} \max \left|\psi^{\prime}\right|\right)^{p+1} \\
\left\|\left(w_{12}-I^{N} w_{12}\right)_{y}\right\|_{0, \Omega_{22}} & \leq C \varepsilon^{1 / 4}\left(N^{-1} \max \left|\psi^{\prime}\right|\right)^{p+1} .
\end{aligned}
$$

We use (3.31) for the $x$-derivative of $w_{2}$ on $\Omega_{12}$ to obtain

$$
\left\|\left(w_{2}-I^{N} w_{2}\right)_{x}\right\|_{0, \Omega_{12}} \leq C\left(h\left\|w_{2 x x}\right\|_{0, \Omega_{12}}+N^{-1}\left\|w_{2 x y}\right\|_{0, \Omega_{12}}\right) \leq C N^{-(p+1)}
$$

while (3.32) gives for the $y$-derivative of $w_{2}$ on $\Omega_{12}$

$$
\left\|\left(w_{2}-I^{N} w_{2}\right)_{y}\right\|_{0, \Omega_{12}} \leq C\left(\left\|w_{2 y}\right\|_{0, \Omega_{12}}+N \varepsilon^{1 / 2} \ln ^{1 / 2} N\left\|w_{2}\right\|_{L_{\infty}\left(\Omega_{12}\right)}\right) \leq C \varepsilon^{1 / 4} N^{-(p+1)} .
$$

For the $y$-derivative of $w_{12}$ on $\Omega_{12}$ and the $x$-derivative of $\Omega_{12}$ on $\Omega_{21}$, we apply (3.32) and obtain

$$
\begin{aligned}
& \left\|\left(w_{12}-I^{N} w_{12}\right)_{y}\right\|_{0, \Omega_{12}} \leq C \varepsilon^{1 / 4} N^{-(p+1)} \\
& \left\|\left(w_{12}-I^{N} w_{12}\right)_{x}\right\|_{0, \Omega_{21}} \leq C \varepsilon^{-1 / 4} N^{-(p+1)} .
\end{aligned}
$$


In order to estimate the remaining two terms, we need the additional Assumption 4. We start with the $x$-derivative of $w_{12}$ on $\Omega_{12}$. The triangle inequality gives

$$
\left\|\left(w_{12}-I^{N} w_{12}\right)_{x}\right\|_{0, \Omega_{12}} \leq\left\|w_{12 x}\right\|_{0, \Omega_{12}}+\left\|\left(I^{N} w_{12}\right)_{x}\right\|_{0, \Omega_{12}} .
$$

The first term can be calculated as usual while applying an inverse inequality and the $L_{\infty}$-stability of $I^{N}$ gives for the second term

$$
\begin{aligned}
\left\|\left(I^{N} w_{12}\right)_{x}\right\|_{0, \Omega_{12}}^{2} & =\sum_{\tau_{i j} \subset \Omega_{12}}\left\|\left(I^{N} w_{12}\right)_{x}\right\|_{0, \tau_{i j}}^{2} \leq C \sum_{\tau_{i j} \subset \Omega_{12}} h_{i}^{-2}\left\|I^{N} w_{12}\right\|_{0, \tau_{i j}}^{2} \\
& \leq C \sum_{\tau_{i j} \subset \Omega_{12}} h_{i}^{-1} k_{j}\left\|I^{N} w_{12}\right\|_{L_{\infty}\left(\tau_{i j}\right)}^{2} \\
& \leq C N h_{\min , \Omega_{12}}^{-1}\left\|I^{N} w_{12}\right\|_{L_{\infty}\left(\Omega_{12}\right)}^{2} \leq C h_{\text {min }, \Omega_{12}}^{-1} N^{-2 \sigma+1} .
\end{aligned}
$$

Due to Assumption 4, the minimal mesh-size is bounded by $h_{\min , \Omega_{12}} \geq C \varepsilon N^{-1}$ and we obtain

$$
\left\|\left(w_{12}-I^{N} w_{12}\right)_{x}\right\|_{0, \Omega_{12}} \leq C \varepsilon^{-1 / 2} N^{-(p+1)}
$$

and similarly

$$
\left\|\left(w_{12}-I^{N} w_{12}\right)_{y}\right\|_{0, \Omega_{21}} \leq C \varepsilon^{-1 / 4} N^{-(p+1)} .
$$

Collecting (3.33)-(3.39) gives

$$
\left\|\nabla\left(u-I^{N} u\right)\right\|_{0} \leq C \varepsilon^{-1 / 2}\left(h+k+N^{-1} \max \left|\psi^{\prime}\right|\right)^{p+1}
$$

and the bound for the energy norm follows.

It remains to prove the estimate (3.26) of the stabilisation term $s$. By (3.21) with $s=0$ and the interpolation error bounds (3.33a) and (3.33c) we obtain

$$
\begin{aligned}
\sum_{\tau \in \Omega_{11} \cup \Omega_{21}} \delta_{\tau}\left\|\kappa_{\tau}\left(b\left(I^{N} v-v\right)_{x}\right)\right\|_{0, \tau}^{2} & \leq C \sum_{\tau \in \Omega_{11} \cup \Omega_{21}} \delta_{\tau}\left\|b\left(I^{N} v-v\right)_{x}\right\|_{0, \tau}^{2} \\
& \leq C\left(\delta_{11}\left\|\left(I^{N} v-v\right)_{x}\right\|_{0, \Omega_{11}}^{2}+\delta_{21}\left\|\left(I^{N} v-v\right)_{x}\right\|_{0, \Omega_{21}}^{2}\right) \\
& \leq C\left(\delta_{11} N^{-2(p+1)}+\delta_{21} \varepsilon^{1 / 2} \ln N\left(k+N^{-1}\right)^{2(p+1)}\right)
\end{aligned}
$$

and (3.26) is proved.

We will now state the main theorems of this paper. We prove superconvergence of the unstabilised Galerkin FEM and the stabilised LPS-FEM in the energy norm and supercloseness of the latter one in the LPS-norm. 
Theorem 13 (Superconvergence Galerkin FEM). We denote by $u$ and $\tilde{u}^{N}$ the solutions of (3.1) and (3.2), respectively and set

$$
C_{\psi}:=1+N^{-1 / 2} \ln ^{1 / 2} N \max \left|\psi^{\prime}\right|
$$

Then, we have

$$
\|\| u-\tilde{u}^{N} \|_{\varepsilon} \leq C C_{\psi}\left(h+k+N^{-1} \max \left|\psi^{\prime}\right|\right)^{p+1} .
$$

Proof. We start with triangle inequality to obtain

$$
\left\|u-\tilde{u}^{N}\left|\left\|\left.\right|_{\varepsilon} \leq\right\| u-I^{N} u\left\|_{\varepsilon}+\right\|\right| I^{N} u-\tilde{u}^{N}\right\| \|_{\varepsilon} .
$$

The first term on the right-hand side is bounded by the interpolation error given in Theorem 12. Applying coercivity (3.6) and Galerkin orthogonality (3.3) to the second term gives

$$
\|\| \|_{\varepsilon}^{2} \leq a_{G a l}(\chi, \chi)=\varepsilon\left(\nabla\left(I^{N} u-u\right), \nabla \chi\right)+\left(c\left(I^{N} u-u\right)-b\left(I^{N} u-u\right)_{x}, \chi\right)
$$

where $\chi:=I^{N} u-\tilde{u}^{N}$. To bound the first term of (3.41), the Cauchy-Schwarz inequality and the enhanced estimates of interpolation error of Theorem 12 are used to achieve

$$
\begin{aligned}
\left|\varepsilon\left(\nabla\left(I^{N} u-u\right), \nabla \chi\right)\right| & \leq \varepsilon\left\|\nabla\left(I^{N} u-u\right)\right\|_{0}\|\nabla \chi\|_{0} \\
& \leq C\left(h+k+N^{-1} \max \left|\psi^{\prime}\right|\right)^{p+1}\|\chi\|_{\varepsilon} .
\end{aligned}
$$

An integration by parts of the second term of (3.41) yields

$$
\left(-b\left(I^{N} u-u\right)_{x}+c\left(I^{N} u-u\right), \chi\right)=\left(I^{N} u-u,\left(c+b_{x}\right) \chi\right)+\left(I^{N} u-u, b \chi_{x}\right) .
$$

We get for the first term on the right-hand side of (3.43)

$$
\left|\left(I^{N} u-u,\left(c+b_{x}\right) \chi\right)\right| \leq C\left\|I^{N} u-u\right\|_{0}\|\chi\|_{0} \leq C\left(h+k+N^{-1} \max \left|\psi^{\prime}\right|\right)^{p+2}\|\| \chi\|\|_{\varepsilon} .
$$

Applying Hölder and Cauchy-Schwarz inequalities to the second term of (3.43) results in

$$
\begin{aligned}
\left|\left(I^{N} u-u, b \chi_{x}\right)\right| \leq C & \left\|I^{N} u-u\right\|_{L_{\infty}\left(\Omega_{12} \cup \Omega_{22}\right)}\left\|\chi_{x}\right\|_{L_{1}\left(\Omega_{12} \cup \Omega_{22}\right)}+ \\
& \left.\left\|I^{N} u-u\right\|_{L_{\infty}\left(\Omega_{21}\right)}\left\|\chi_{x}\right\|_{L_{1}\left(\Omega_{21}\right)}+\left\|I^{N} u-u\right\|_{0, \Omega_{11}}\left\|\chi_{x}\right\|_{0, \Omega_{11}}\right) .
\end{aligned}
$$

With meas $\left(\Omega_{12} \cup \Omega_{22}\right) \leq C \varepsilon \ln N$, it follows

$$
\begin{aligned}
\left\|I^{N} u-u\right\|_{L_{\infty}\left(\Omega_{12} \cup \Omega_{22}\right)}\left\|\chi_{x}\right\|_{L_{1}\left(\Omega_{12} \cup \Omega_{22}\right)} & \\
& \leq C\left(h+k+N^{-1} \max \left|\psi^{\prime}\right|\right)^{p+2} \ln ^{1 / 2} N \mid\|\chi\| \|_{\varepsilon} \\
& \leq C N^{-1} \max \left|\psi^{\prime}\right| \ln ^{1 / 2} N\left(h+k+N^{-1} \max \left|\psi^{\prime}\right|\right)^{p+1}\||\chi|\|_{\varepsilon} .
\end{aligned}
$$


Using an inverse inequality, we obtain on $\Omega_{21}$

$$
\varepsilon^{1 / 4} N^{-1 / 2}\left\|\chi_{x}\right\|_{0, \Omega_{21}}=\left(\left(\varepsilon^{1 / 2}\left\|\chi_{x}\right\|_{0, \Omega_{21}}\right)\left(N^{-1}\left\|\chi_{x}\right\|_{0, \Omega_{21}}\right)\right)^{1 / 2} \leq C\|\| \chi \|_{\varepsilon} .
$$

Thus, the estimate

$$
\begin{aligned}
\left\|I^{N} u-u\right\|_{L_{\infty}\left(\Omega_{21}\right)} & \left\|\chi_{x}\right\|_{L_{1}\left(\Omega_{21}\right)} \\
& \leq C\left(k+N^{-1} \max \left|\psi^{\prime}\right|\right)^{p+1}\left(k+N^{-1} \max \left|\psi^{\prime}\right|\right) \varepsilon^{1 / 4} \ln ^{1 / 2} N\left\|\chi_{x}\right\|_{0, \Omega_{21}} \\
& \leq C\left(k+N^{-1} \max \left|\psi^{\prime}\right|\right)^{p+1}\left(1+N^{-1 / 2} \max \left|\psi^{\prime}\right| \ln ^{1 / 2} N\right)\|\chi\| \|_{\varepsilon}
\end{aligned}
$$

is obtained. Finally, an inverse inequality is used on $\Omega_{11}$. Altogether, we obtain

$$
\left|\left(I^{N} u-u, b \chi_{x}\right)\right| \leq C\left(1+N^{-1 / 2} \max \left|\psi^{\prime}\right| \ln ^{1 / 2} N\right)\left(h+k+N^{-1} \max \left|\psi^{\prime}\right|\right)^{p+1}\||\chi|\|_{\varepsilon} .
$$

Combining (3.41)-(3.45) with the interpolation error of Theorem 12 finishes the proof.

Remark 14. The factor $C_{\psi}$ defined in (3.40) is bounded by a constant for all meshes considered in Table 1.

Nevertheless, S-type meshes fulfilling Assumption \& and $\max \left|\psi^{\prime}\right|=C N$ can be constructed, e.g. with $\xi_{0}:=\frac{1}{2}\left(1-N^{-1} \ln N\right)$

$$
\phi= \begin{cases}\left(N+\frac{2 \ln N-N}{1-N^{-1} \ln N}\right) t, & 0 \leq t \leq \xi_{0} \\ N\left(t-\frac{1}{2}\right)+\ln N, & \xi_{0} \leq t \leq \frac{1}{2}\end{cases}
$$

Here $\max \left|\psi^{\prime}\right| \geq\left|\psi^{\prime}\left(\xi_{0}+\right)\right|=\phi^{\prime}\left(\xi_{0}+\right) \mathrm{e}^{-\phi\left(\xi_{0}\right)}=N e^{-1}$. For this type of meshes, $C_{\psi}$ will increase with increasing $N$.

Theorem 15 (Superconvergence LPS-FEM). The solutions of (3.1) and (3.5) are denoted by $u$ and $u^{N}$, respectively. Furthermore, the stabilisation parameters are chosen according to

$$
\begin{aligned}
& \delta_{11} \leq C N^{-2}\left(\max \left|\psi^{\prime}\right|\right)^{2(p+1)}, \\
& \delta_{21} \leq C \varepsilon^{-1 / 2} \ln ^{-1} N\left(k+N^{-1} \max \left|\psi^{\prime}\right|\right)^{2}, \\
& \delta_{12}=\delta_{22}=0 .
\end{aligned}
$$

Then, we have

$$
\left\|u-u^{N}\right\|_{\varepsilon} \leq C C_{\psi}\left(h+k+N^{-1} \max \left|\psi^{\prime}\right|\right)^{p+1}
$$

and

$$
\left\|\left|u^{I}-u^{N}\right|\right\|_{L P S} \leq C C_{\psi}\left(h+k+N^{-1} \max \left|\psi^{\prime}\right|\right)^{p+1}
$$

with $C_{\psi}$ defined by (3.40). 
Proof. We start with triangle inequality to obtain

$$
\left.\left\|u-u^{N}\right\|\right|_{\varepsilon} \leq\left\|u-I^{N} u\right\|_{\varepsilon}+\left\|\mid I^{N} u-u^{N}\right\|_{\varepsilon} .
$$

The first term on the right-hand side is bounded by Theorem 12. For the second term, we estimate by

$$
\left\|\mid I^{N} u-u^{N}\right\|_{\varepsilon} \leq\|\| I^{N} u-u^{N} \|_{L P S} .
$$

Thus, (3.47) is a consequence of (3.48) that will be proven now. We apply coercivity (3.6) and weak Galerkin orthogonality (3.7) to get

$$
\begin{aligned}
\left.\left\|I^{N} u-u^{N}\right\|\right|_{L P S} ^{2} & \leq a_{L P S}\left(I^{N} u-u, I^{N} u-u^{N}\right) \\
& \leq a_{G a l}\left(I^{N} u-u, \chi\right)+s\left(I^{N} u, \chi\right)
\end{aligned}
$$

where $\chi:=I^{N} u-u^{N}$. The first term of (3.49) can be bounded as in the proof of Theorem 13. Now only the stabilisation term $s\left(I^{N} u, \chi\right)$ stays to be estimated. Bounds for $s(w, \chi)$ for an arbitrary function $w$ are derived by

$$
|s(w, \chi)| \leq\left(\sum_{\tau \in \Omega_{11} \cup \Omega_{21}} \delta_{\tau}\left\|\kappa_{\tau}\left(b w_{x}\right)\right\|_{0, \tau}^{2}\right)^{1 / 2}\|\chi\| \|_{L P S} .
$$

Thus, the occurring sums must be estimated. We start with the non-layer function $v$. Expanding the stabilisation term yields

$$
s\left(I^{N} v, \chi\right)=s\left(I^{N} v-v, \chi\right)+s(v, \chi) .
$$

We apply (3.21) with $s=p$ to the second term on the right-hand side of (3.50) and obtain

$$
\begin{aligned}
\sum_{\tau \in \Omega_{11} \cup \Omega_{21}} \delta_{\tau}\left\|\kappa_{\tau}\left(b v_{x}\right)\right\|_{0, \tau}^{2} & \leq C \sum_{\tau_{i j} \in \Omega_{11} \cup \Omega_{21}} \delta_{\tau_{i j}} \sum_{r=0}^{p} h_{i}^{2(p-r)} k_{j}^{2 r}\left\|\frac{\partial^{p+1} v}{\partial x^{p-r+1} \partial y^{r}}\right\|_{0, \tau_{i j}}^{2} \\
& \leq C\left(\delta_{11} N^{-2 p}+\delta_{21} \varepsilon^{1 / 2} \ln N\left(k+N^{-1}\right)^{2 p}\right) .
\end{aligned}
$$

Combining this estimate with (3.26) gives

$$
\left|s\left(I^{N} v, \chi\right)\right| \leq C\left(\delta_{11} N^{-2 p}+\delta_{21} \varepsilon^{1 / 2} \ln N\left(k+N^{-1}\right)^{2 p}\right)^{1 / 2}\|\| \chi \|_{L P S} .
$$

Due to the bounds given in Assumption 1, we can estimate

$$
\begin{aligned}
\left\|I^{N}\left(w_{1}+w_{12}\right)\right\|_{0, \Omega_{21}}^{2} & \leq \operatorname{meas}\left(\Omega_{21}\right)\left\|I^{N}\left(w_{1}+w_{12}\right)\right\|_{L_{\infty}\left(\Omega_{21}\right)}^{2} \\
& \leq C \operatorname{meas}\left(\Omega_{21}\right)\left\|w_{1}+w_{12}\right\|_{L_{\infty}\left(\Omega_{21}\right)}^{2} \leq C \varepsilon^{1 / 2} \ln N N^{-2 \sigma}
\end{aligned}
$$


where the $L_{\infty}$-stability of the interpolation operator $I^{N}$ was exploited. In order to estimate $I^{N}\left(w_{1}+w_{12}\right)$ on $\Omega_{11}$, we calculate for $w=w_{1}+w_{12}$ :

$$
\begin{aligned}
\left\|I^{N} w\right\|_{0, \Omega_{11}}^{2} & =\sum_{i=N / 2+1}^{N} \int_{x_{i-1}}^{x_{i}} \int_{\lambda_{y}}^{1}\left|\left(I^{N} w\right)(x, y)\right|^{2} d y d x \\
& \leq C \sum_{i=N / 2+2}^{N} \int_{x_{i-1}}^{x_{i}} \mathrm{e}^{-2 \beta x_{i-1} / \varepsilon} d x+C \int_{x_{i-1}}^{x_{i}} \mathrm{e}^{-2 \beta x_{N / 2} / \varepsilon} d x \\
& \leq C\left(\varepsilon+N^{-1}\right) N^{-2 \sigma}
\end{aligned}
$$

where we have used Assumption 1, the monotonicity of the exponential functions, and the definition of $\lambda_{x}=x_{N / 2}$. Thus, applying (3.21) with $s=0$ gives for $w=w_{1}+w_{12}$

$$
\begin{aligned}
\sum_{\tau \in \Omega_{11} \cup \Omega_{21}} \delta_{\tau}\left\|\kappa_{\tau}\left(b\left(I^{N} w\right)_{x}\right)\right\|_{0, \tau}^{2} & \leq C \sum_{\tau \in \Omega_{11} \cup \Omega_{21}} \delta_{\tau}\left\|\left(I^{N} w\right)_{x}\right\|_{0, \tau}^{2} \leq C \sum_{\tau \in \Omega_{11} \cup \Omega_{21}} \delta_{\tau} N^{2}\left\|I^{N} w\right\|_{0, \tau}^{2} \\
& \leq C\left(\delta_{11}\left(N^{-1}+\varepsilon\right)+\delta_{21} \varepsilon^{1 / 2} \ln N\right) N^{-2(\sigma-1)} .
\end{aligned}
$$

Therefore, the estimate

$$
\left|s\left(I^{N}\left(w_{1}+w_{12}\right), \chi\right)\right| \leq C\left(\delta_{11}\left(N^{-1}+\varepsilon\right)+\delta_{21} \varepsilon^{1 / 2} \ln N\right)^{1 / 2} N^{-(p+1)}\|\| \chi \|_{L P S}
$$

holds true. We get similarly for $w_{2}$ on $\Omega_{11}$

$$
\sum_{\tau \in \Omega_{11}} \delta_{\tau}\left\|\kappa_{\tau}\left(b\left(I^{N} w_{2}\right)_{x}\right)\right\|_{0, \tau}^{2} \leq C \sum_{\tau \in \Omega_{11}} \delta_{\tau} N^{2}\left\|I^{N} w_{2}\right\|_{0, \tau}^{2} \leq C \delta_{11}\left(N^{-1}+\varepsilon^{1 / 2}\right) N^{-2(\sigma-1)}
$$

while we proceed on $\Omega_{21}$ as done for $v$ and use (2.6) to get

$$
\begin{aligned}
\sum_{\tau \in \Omega_{21}} \delta_{\tau}\left\|\kappa_{\tau}\left(b\left(w_{2}\right)_{x}\right)\right\|_{0, \tau}^{2} & \leq C \sum_{\tau_{i j} \in \Omega_{21}} \delta_{\tau} \sum_{r=0}^{p} N^{-2(p-r)}\left\|k_{j}^{r} \frac{\partial^{p+1} w_{2}}{\partial x^{p-r+1} \partial y^{r}}\right\|_{0, \tau_{i j}}^{2} \\
& \leq C \delta_{21} \varepsilon^{1 / 2}\left(N^{-1} \max \left|\psi^{\prime}\right|\right)^{2 p}
\end{aligned}
$$

and

$$
\begin{aligned}
\sum_{\tau \in \Omega_{21}} \delta_{\tau}\left\|\kappa_{\tau}\left(b\left(I^{N} w_{2}-w_{2}\right)_{x}\right)\right\|_{0, \tau}^{2} & \leq C \delta_{21}\left\|\left(I^{N} w_{2}-w_{2}\right)_{x}\right\|_{0, \Omega_{21}}^{2} \\
& \leq C \delta_{21} \varepsilon^{1 / 2}\left(N^{-1} \max \left|\psi^{\prime}\right|\right)^{2(p+1)}
\end{aligned}
$$

Hence, we get

$$
\begin{aligned}
& \left|s\left(I^{N} w_{2}, \chi\right)\right| \\
& \quad \leq C\left(\delta_{11}\left(N^{-1}+\varepsilon^{1 / 2}\right) N^{-2(p+1)}+\delta_{21} \varepsilon^{1 / 2}\left(N^{-1} \max \left|\psi^{\prime}\right|\right)^{2 p}\right)^{1 / 2}\|\| \chi \|_{L P S} .
\end{aligned}
$$


Combining (3.51)-(3.53) gives

$$
\begin{aligned}
& \left|s\left(I^{N} u, \chi\right)\right| \\
& \quad \leq C\left(\delta_{11}^{1 / 2} N^{-p}+\left(\delta_{21} \varepsilon^{1 / 2}\right)^{1 / 2}\left(\ln ^{1 / 2} N\left(k+N^{-1}\right)^{p}+\left(N^{-1} \max \left|\psi^{\prime}\right|\right)^{p}\right)\right)\||\chi|\|_{L P S} .
\end{aligned}
$$

With (3.46), it follows

$$
\left|s\left(I^{N} u, \chi\right)\right| \leq C\left(k+N^{-1} \max \left|\psi^{\prime}\right|\right)^{p+1} \mid\|\chi\|_{L P S}
$$

and we are done.

Remark 16. The orthogonality property (3.18) was not used in the proof of Theorem 15. It can be applied to estimate the second term of (3.43) on $\Omega_{11} \cup \Omega_{21}$ as follows

$$
\begin{aligned}
\left(I^{N} u-u, b \chi_{x}\right)_{\Omega_{11} \cup \Omega_{21}} & =\sum_{\tau \in \Omega_{11} \cup \Omega_{21}}\left(I^{N} u-u, b \chi_{x}-\pi_{\tau}\left(b \chi_{x}\right)\right)_{\tau} \\
& \leq C\left(\sum_{\tau \in \Omega_{11} \cup \Omega_{21}} \delta_{\tau}^{-1}\left\|I^{N} u-u\right\|_{0, \tau}^{2}\right)^{1 / 2}\|\| \chi \|_{L P S} \\
& \leq C\left(\delta_{11}^{-1} N^{-2(p+2)}+\delta_{21}^{-1}\left(N^{-1} \max \left|\psi^{\prime}\right|\right)^{2(p+2)}\right)^{1 / 2}\|\chi\| \|_{L P S} .
\end{aligned}
$$

If

$$
\delta_{11} \geq C N^{-2}\left(\max \left|\psi^{\prime}\right|\right)^{2(p+1)}, \quad \delta_{21} \geq C\left(k+N^{-1} \max \left|\psi^{\prime}\right|\right)^{2},
$$

we obtain

$$
\left|\left(I^{N} u-u, b \chi_{x}\right)_{\Omega_{11} \cup \Omega_{21}}\right| \leq C\left(h+k+N^{-1} \max \left|\psi^{\prime}\right|\right)^{p+1}\left|\|\chi \mid\|_{L P S}\right.
$$

and (3.40) can be replaced by the larger

$$
C_{\psi}:=1+N^{-1} \ln ^{1 / 2} N \max \left|\psi^{\prime}\right|
$$

Comparing the bounds (3.54) and (3.46) for $\delta_{11}$ and $\delta_{21}$ shows

$$
\begin{aligned}
& C N^{-2}\left(\max \left|\psi^{\prime}\right|\right)^{2(p+1)} \leq \delta_{11} \leq C N^{-2}\left(\max \left|\psi^{\prime}\right|\right)^{2(p+1)}, \\
& C\left(k+N^{-1} \max \left|\psi^{\prime}\right|\right)^{2} \leq \delta_{21} \leq C \varepsilon^{-1 / 2} \ln ^{-1} N\left(k+N^{-1} \max \left|\psi^{\prime}\right|\right)^{2} .
\end{aligned}
$$

Thus, $\delta_{11}$ is fixed up to a constant factor. Recall, $\varepsilon^{-1 / 2} \ln ^{-1} N \geq 4 \sigma \geq 16$ due to (2.2), $\sigma \geq p+2$, and $p \geq 2$.

\section{Numerical results}

We consider the singularly perturbed convection-diffusion problem

$$
\begin{aligned}
-\varepsilon \Delta u-(2-x) u_{x}+\frac{3}{2} u & =f & & \text { in } \Omega=(0,1)^{2}, \\
u & =0 & & \text { on } \partial \Omega,
\end{aligned}
$$


where the right-hand side $f$ of (4.1) was chosen such that

$$
u(x, y)=\left(\cos \frac{\pi x}{2}-\frac{\mathrm{e}^{-x / \varepsilon}-\mathrm{e}^{-1 / \varepsilon}}{1-\mathrm{e}^{-1 / \varepsilon}}\right) \frac{\left(1-\mathrm{e}^{-y / \sqrt{\varepsilon}}\right)\left(1-\mathrm{e}^{-(1-y) / \sqrt{\varepsilon}}\right)}{1-\mathrm{e}^{-1 / \sqrt{\varepsilon}}}
$$

is the solution of (4.1). This problem was taken from [13]. The function $u$ shows an exponential boundary layer at $x=0$ and two characteristic boundary layers at $y=0$ and $y=1$, respectively. Moreover, Assumption 1 is satisfied.

All calculations were carried out using the program package MooNMD [15]. The obtained systems of linear equations were solved directly by UMFPACK [7-10].

In the following, 'ord' will denote always the exponent $\alpha$ in a convergence order of form $\mathcal{O}\left(N^{-\alpha}\right)$ while 'ln-ord' corresponds to the exponent $\alpha$ in a convergence order of form $\mathcal{O}\left(\left(N^{-1} \ln N\right)^{\alpha}\right)$.

\subsection{The bilinear case}

We will have first a look at the results obtained for bilinear finite elements. All error norms were calculated by using a $3 \times 3$ Gaussian quadrature formula. We have chosen $\sigma=5 / 2$.

Table 2: Galerkin, $Q_{1}, \varepsilon=10^{-8}$.

\begin{tabular}{r|ccc|cc|ccc|cc} 
& \multicolumn{5}{|c|}{$\| u^{I}-\tilde{u}^{N}||_{\varepsilon}$} & \multicolumn{5}{c}{$\left\|u-\tilde{u}^{N}\right\|_{\varepsilon}$} \\
& \multicolumn{3}{|c}{ S-mesh } & \multicolumn{2}{c|}{ B-S mesh } & \multicolumn{3}{c}{ S-mesh } & \multicolumn{2}{c}{ B-S mesh } \\
$N$ & error & ord & ln-ord & error & ord & error & ord & ln-ord & error & ord \\
\hline 4 & $2.467-1$ & & & $1.326-1$ & & $3.819-1$ & & & $2.644-1$ & \\
8 & $1.104-1$ & 1.16 & 1.98 & $3.274-2$ & 2.02 & $2.683-1$ & 0.51 & 0.87 & $1.465-1$ & 0.85 \\
16 & $4.991-2$ & 1.15 & 1.69 & $8.706-3$ & 1.91 & $1.777-1$ & 0.59 & 0.88 & $7.756-2$ & 0.92 \\
32 & $1.987-2$ & 1.33 & 1.80 & $2.291-3$ & 1.93 & $1.107-1$ & 0.68 & 0.93 & $3.994-2$ & 0.96 \\
64 & $7.212-3$ & 1.46 & 1.88 & $5.931-4$ & 1.95 & $6.637-2$ & 0.74 & 0.95 & $2.027-2$ & 0.98 \\
128 & $2.459-3$ & 1.55 & 1.92 & $1.517-4$ & 1.97 & $3.870-2$ & 0.78 & 0.96 & $1.021-2$ & 0.99 \\
256 & $8.027-4$ & 1.62 & 1.95 & $3.847-5$ & 1.98 & $2.211-2$ & 0.81 & 0.97 & $5.127-3$ & 0.99 \\
512 & $2.538-4$ & 1.66 & 1.96 & $9.707-6$ & 1.99 & $1.244-2$ & 0.83 & 0.98 & $2.569-3$ & 1.00 \\
1024 & $7.827-5$ & 1.70 & 1.97 & $2.440-6$ & 1.99 & $6.909-3$ & 0.85 & 0.98 & $1.286-3$ & 1.00
\end{tabular}

Tab. 2 presents for the standard Galerkin method the numerical results for the perturbation parameter $\varepsilon=10^{-8}$. The errors $\left\|\left|u^{I}-\tilde{u}^{N}\right|\right\|_{\varepsilon}$ and \|\|$u-\tilde{u}^{N} \mid\|\|_{\varepsilon}$ are shown for calculations on S-meshes and B-S meshes. Here, $u^{I}$ denotes the nodal bilinear interpolant of the solution $u$ and $\tilde{u}^{N}$ is the Galerkin solution. The errors converge with the predicted orders, compare Lemma 7 . We clearly see that the typical logarithmic factor is present only the classical S-mesh. Moreover, the errors on B-S meshes are smaller than those on $\mathrm{S}$-meshes. The errors in the $\varepsilon$-weighted energy norm given in Tab. 2 indicate clearly that 
Table 3: Galerkin, $Q_{1}, N=1024$.

\begin{tabular}{c|c|c|c|c} 
& \multicolumn{2}{|c|}{$\left\|\mid u^{I}-\tilde{u}^{N}\right\|_{\varepsilon}$} & \multicolumn{2}{|c}{$\left\|u-\tilde{u}^{N}\right\| \|_{\varepsilon}$} \\
$\varepsilon$ & S-mesh & B-S mesh & S-mesh & B-S mesh \\
\hline $10^{-2}$ & $6.566279-5$ & $1.822452-4$ & $5.817575-3$ & $1.289396-3$ \\
$10^{-3}$ & $7.575957-5$ & $1.148876-5$ & $6.667492-3$ & $1.658155-3$ \\
$10^{-4}$ & $8.334820-5$ & $2.992066-6$ & $6.943117-3$ & $1.291919-3$ \\
$10^{-5}$ & $7.970112-5$ & $2.429980-6$ & $6.919573-3$ & $1.287513-3$ \\
$10^{-6}$ & $7.863484-5$ & $2.375848-6$ & $6.912102-3$ & $1.286122-3$ \\
$10^{-7}$ & $7.837084-5$ & $2.443810-6$ & $6.909738-3$ & $1.285682-3$ \\
$10^{-8}$ & $7.826662-5$ & $2.440353-6$ & $6.908990-3$ & $1.285544-3$ \\
$10^{-9}$ & $7.823014-5$ & $2.435213-6$ & $6.908754-3$ & $1.285500-3$ \\
$10^{-10}$ & $7.821823-5$ & $2.433162-6$ & $6.908679-3$ & $1.285486-3$ \\
$10^{-11}$ & $7.821443-5$ & $2.432471-6$ & $6.908655-3$ & $1.285481-3$ \\
$10^{-12}$ & $7.821323-5$ & $2.432248-6$ & $6.908648-3$ & $1.285480-3$
\end{tabular}

the standard Galerkin method convergences with first order only while a supercloseness results of second order holds.

The errors $\left\|\left|u^{I}-\tilde{u}^{N}\right|\right\|_{\varepsilon}$ and $\left\|\mid u-\tilde{u}^{N}\right\|_{\varepsilon}$ for the fixed value $N=1024$ and $\varepsilon \in$ $\left\{10^{-2}, 10^{-3}, \ldots, 10^{-12}\right\}$ are given in Tab. 3 . The robustness of the errors with respect to $\varepsilon \rightarrow 0$ can be perceived.

Table 4: LPS, $Q_{1}, \varepsilon=10^{-8}$.

\begin{tabular}{r|ccc|cc|ccc|cc} 
& \multicolumn{5}{|c|}{$\| u^{I}-u^{N}||_{L P S}$} & \multicolumn{5}{c}{$\left\|u-u^{N}\right\|_{L P S}$} \\
& \multicolumn{3}{|c|}{ S-mesh } & \multicolumn{2}{c|}{ B-S mesh } & \multicolumn{3}{c}{ S-mesh } & \multicolumn{2}{c}{ B-S mesh } \\
$N$ & error & ord & ln-ord & error & ord & error & ord & ln-ord & error & ord \\
\hline 4 & $2.466-1$ & & & $1.325-1$ & & $3.829-1$ & & & $2.652-1$ & \\
8 & $1.104-1$ & 1.16 & 1.98 & $3.274-2$ & 2.02 & $2.685-1$ & 0.51 & 0.88 & $1.465-1$ & 0.86 \\
16 & $4.991-2$ & 1.15 & 1.69 & $8.706-3$ & 1.91 & $1.777-1$ & 0.60 & 0.88 & $7.756-2$ & 0.92 \\
32 & $1.987-2$ & 1.33 & 1.80 & $2.291-3$ & 1.93 & $1.108-1$ & 0.68 & 0.93 & $3.994-2$ & 0.96 \\
64 & $7.212-3$ & 1.46 & 1.88 & $5.931-4$ & 1.95 & $6.637-2$ & 0.74 & 0.95 & $2.027-2$ & 0.98 \\
128 & $2.459-3$ & 1.55 & 1.92 & $1.517-4$ & 1.97 & $3.870-2$ & 0.78 & 0.96 & $1.021-2$ & 0.99 \\
256 & $8.027-4$ & 1.62 & 1.95 & $3.847-5$ & 1.98 & $2.211-2$ & 0.81 & 0.97 & $5.127-3$ & 0.99 \\
512 & $2.538-4$ & 1.66 & 1.96 & $9.707-6$ & 1.99 & $1.244-2$ & 0.83 & 0.98 & $2.569-3$ & 1.00 \\
1024 & $7.827-5$ & 1.68 & 1.97 & $2.440-6$ & 1.99 & $6.909-3$ & 0.85 & 0.98 & $1.286-3$ & 1.00
\end{tabular}

Tab. 4 shows the errors $\left\|\left|u^{I}-u^{N}\right|\right\|_{L P S}$ and $\left\|\mid u-u^{N}\right\| \|_{L P S}$ for the local projection method where $\varepsilon=10^{-8}$ was used. The stabilisation parameters $\delta_{11}$ and $\delta_{21}$ were chosen to equal the upper bounds given in Theorem 8 with $C=1$. The error $\left\|\left|u^{I}-u^{N}\right|\right\|_{L P S}$ 
converges with the predicted order 1, compare Theorem 8. Instead of showing the error $\left\|\left|u-u^{N}\right|\right\|_{\varepsilon}$ in the $\varepsilon$-weighted energy norm, we present the error $\left\|\left|u-u^{N}\right|\right\|_{L P S}$ in the local projection norm which also converges with first order. Note again that the logarithmic factor is only present on S-meshes and that the error norms on B-S meshes are smaller than those on S-meshes. As for the standard Galerkin method, the local projection scheme shows only first order convergence but provides a supercloseness result of second order.

\subsection{The higher order case}

Let us now turn to discretisations with higher order elements. We will show results of calculations with the enriched element $\mathcal{Q}_{2}^{+}$, i.e., $p=2$. Furthermore, we have chosen $\sigma=4$. In the following, all error norms were calculated by applying a $5 \times 5$ Gaussian quadrature formula.

Table 5: Galerkin, $Q_{2}^{+}, \varepsilon=10^{-8},\left\|u-\tilde{u}^{N}\right\|_{\varepsilon}$.

\begin{tabular}{r|ccc|cc} 
& \multicolumn{3}{|c|}{ S-mesh } & \multicolumn{2}{c}{ B-S mesh } \\
$N$ & error & ord & ln-ord & error & ord \\
\hline 4 & $3.444-2$ & & & $1.602-2$ & \\
8 & $1.637-2$ & 1.07 & 1.83 & $3.099-3$ & 2.37 \\
16 & $5.397-3$ & 1.60 & 2.36 & $4.673-4$ & 2.73 \\
32 & $1.397-3$ & 1.95 & 2.65 & $6.382-5$ & 2.87 \\
64 & $3.093-4$ & 2.17 & 2.80 & $8.339-6$ & 2.94 \\
128 & $6.199-5$ & 2.32 & 2.87 & $1.066-6$ & 2.97 \\
256 & $1.161-5$ & 2.42 & 2.91 & $1.348-7$ & 2.98
\end{tabular}

Tab. 5 gives the error $\left\|\left|u-\tilde{u}^{N}\right|\right\|_{\varepsilon}$ for the standard Galerkin method on S-meshes and $\mathrm{B}-\mathrm{S}$ meshes where we have chosen $\varepsilon=10^{-8}$. The obtained convergence orders correspond to the ones predicted by Theorem 13. As for the lowest order elements, the logarithmic factor is present only on S-meshes. Furthermore, the results on B-S meshes are much smaller than those on S-meshes. The difference in the local projection norm is almost two orders of magnitude on the finest mesh with $N=256$.

The errors $\left\|\left|u-u^{N}\right|\right\|_{L P S}$ and $\left\|\mid I^{N} u-u^{N}\right\|_{L P S}$ for local projection method are shown in Tab. 6. We have chosen $\varepsilon=10^{-8}$ in our calculations on S-meshes and B-S meshes. The stabilisation parameters $\delta_{11}$ and $\delta_{21}$ were set to the upper bounds given in (3.46) with $C=0.01$. For larger values of $C$, the stabilisation term $s$ dominates the complete discretisation and worse results are obtained. Although third order convergence is proved only for $\left\|\left|I^{N} u-u^{N}\right|\right\|_{L P S}$ and \|\|$u-I^{N} u\|\|_{\varepsilon}$, also $\left\|u-u^{N}\right\| \|_{L P S}$ converges with third order. From the results in Tab. 6, it is again obvious that the results on B-S meshes are much smaller than the corresponding results on S-meshes. On both types of meshes, the convergence order given in Theorem 15 is achieved. 
Table 6: LPS, $Q_{2}^{+}, \varepsilon=10^{-8}$.

\begin{tabular}{r|ccc|cc|ccc|cc} 
& \multicolumn{5}{|c|}{$\left.\left\|u-u^{N}\right\|\right|_{L P S}$} & \multicolumn{5}{c}{$\left\|I^{N} u-u^{N}\right\|_{L P S}$} \\
\multicolumn{1}{|c|}{} & \multicolumn{3}{|c|}{ S-mesh } & \multicolumn{2}{c|}{ B-S mesh } & \multicolumn{3}{|c}{ S-mesh } & \multicolumn{2}{c}{ B-S mesh } \\
$N$ & error & ord & ln-ord & error & ord & error & ord & ln-ord & error & ord \\
\hline 4 & $5.957-2$ & & & $3.180-2$ & & $5.797 \mathrm{e}-02$ & & & $3.176 \mathrm{e}-02$ & \\
8 & $2.643-2$ & 1.17 & 2.00 & $4.353-3$ & 2.87 & $2.287 \mathrm{e}-02$ & 1.34 & 2.29 & $3.343 \mathrm{e}-03$ & 3.25 \\
16 & $7.970-3$ & 1.73 & 2.55 & $5.343-4$ & 3.03 & $6.211 \mathrm{e}-03$ & 1.88 & 2.77 & $2.847 \mathrm{e}-04$ & 3.55 \\
32 & $1.841-3$ & 2.11 & 2.87 & $6.894-5$ & 2.95 & $1.251 \mathrm{e}-03$ & 2.31 & 3.14 & $2.902 \mathrm{e}-05$ & 3.29 \\
64 & $3.808-4$ & 2.27 & 2.92 & $8.755-6$ & 2.98 & $2.311 \mathrm{e}-04$ & 2.44 & 3.13 & $3.062 \mathrm{e}-06$ & 3.24 \\
128 & $7.433-5$ & 2.36 & 2.92 & $1.094-6$ & 3.00 & $4.267 \mathrm{e}-05$ & 2.44 & 3.02 & $3.062 \mathrm{e}-07$ & 3.32 \\
256 & $1.367-5$ & 2.44 & 2.94 & $1.361-7$ & 3.01 & $7.530 \mathrm{e}-06$ & 2.50 & 3.01 & $2.979 \mathrm{e}-08$ & 3.36
\end{tabular}

Table 7: $Q_{2}^{+}, N=256$.

\begin{tabular}{c|cc|cc} 
& \multicolumn{2}{|c|}{ Galerkin: $\left\|u-\tilde{u}^{N} \mid\right\|_{\varepsilon}$} & \multicolumn{2}{|c}{ LPS: $\left\|\mid u-u^{N}\right\| \|_{L P S}$} \\
$\varepsilon$ & S-mesh & B-S mesh & S-mesh & B-S mesh \\
\hline $10^{-2}$ & $9.682492-6$ & $1.232832-7$ & $1.012785-5$ & $1.232858-7$ \\
$10^{-3}$ & $1.103331-5$ & $7.556524-7$ & $1.226191-5$ & $7.556633-7$ \\
$10^{-4}$ & $1.465276-5$ & $1.705235-7$ & $1.578599-5$ & $1.705462-7$ \\
$10^{-5}$ & $1.262721-5$ & $1.467728-7$ & $1.440307-5$ & $1.468317-7$ \\
$10^{-6}$ & $1.191492-5$ & $1.384128-7$ & $1.386987-5$ & $1.385962-7$ \\
$10^{-7}$ & $1.168062-5$ & $1.356619-7$ & $1.371423-5$ & $1.361917-7$ \\
$10^{-8}$ & $1.160555-5$ & $1.347803-7$ & $1.367229-5$ & $1.361042-7$ \\
$10^{-9}$ & $1.158171-5$ & $1.345003-7$ & $1.366689-5$ & $1.372494-7$ \\
$10^{-10}$ & $1.157416-5$ & $1.344117-7$ & $1.368327-5$ & $1.391335-7$ \\
$10^{-11}$ & $1.157177-5$ & $1.343836-7$ & $1.373989-5$ & $1.408544-7$ \\
$10^{-12}$ & $1.157101-5$ & $1.343748-7$ & $1.389567-5$ & $1.418745-7$
\end{tabular}

The errors $\left\|\mid u-\tilde{u}^{N}\right\| \|_{\varepsilon}$ for the Galerkin method and the error $\left\|u-u^{N}\right\| \|_{L P S}$ for the local projection method are shown in Tab. 7 for the fixed value $N=256$ and $\varepsilon \in$ $\left\{10^{-2}, 10^{-3}, \ldots, 10^{-12}\right\}$. It can be seen that both methods are robust with respect to $\varepsilon \rightarrow 0$ although there seems to be a slight increase of the error for the local projection method. 


\section{References}

[1] T. Apel. Anisotropic finite elements: local estimates and applications. Advances in Numerical Mathematics. B. G. Teubner, Stuttgart, 1999.

[2] N. S. Bakhvalov. The optimization of methods of solving boundary value problems with a boundary layer. U.S.S.R. Comput. Math. Math. Phys., 9(4):139-166, 1969.

[3] R. Becker and M. Braack. A finite element pressure gradient stabilization for the Stokes equations based on local projections. Calcolo, 38(4):173-199, 2001.

[4] R. Becker and M. Braack. A two-level stabilization scheme for the Navier-Stokes equations. In M. Feistauer, V. Dolejší, P. Knobloch, and K. Najzar, editors, Numerical mathematics and advanced applications, pages 123-130, Berlin, 2004. SpringerVerlag.

[5] M. Braack and E. Burman. Local projection stabilization for the Oseen problem and its interpretation as a variational multiscale method. SIAM J. Numer. Anal., 43(6):2544-2566, 2006.

[6] S. C. Brenner and L. R. Scott. The mathematical theory of finite element methods, volume 15 of Texts in Applied Mathematics. Springer-Verlag, New York, second edition, 2002.

[7] T. A. Davis. Algorithm 832: UMFPACK V4.3 - an unsymmetric-pattern multifrontal method. ACM Trans. Math. Software, 30(2):196-199, 2004.

[8] T. A. Davis. A column pre-ordering strategy for the unsymmetric-pattern multifrontal method. ACM Trans. Math. Software, 30(2):167-195, 2004.

[9] T. A. Davis and I. S. Duff. An unsymmetric-pattern multifrontal method for sparse LU factorization. SIAM J. Matrix Anal. Appl., 18(1):140-158, 1997.

[10] T. A. Davis and I. S. Duff. A combined unifrontal/multifrontal method for unsymmetric sparse matrices. ACM Trans. Math. Software, 25(1):1-20, 1999.

[11] S. Franz. Continuous interior penalty method on a shishkin mesh for convectiondiffusion problems with characteristic boundary layers. Comput. Meth. Appl. Mech. Engng., 197(45-48):3679-3686, 2008.

[12] S. Franz. Singularly perturbed problems with characteristic layers: Supercloseness and postprocessing. PhD thesis, TU Dresden, 2008. urn:nbn:de:bsz:14-ds-121862956625173654 .

[13] S. Franz, T. Linß, and H.-G. Roos. Superconvergence analysis of the sdfem for elliptic problems with characteristic layers. Appl. Numer. Math., DOI: 10.1016/j.apnum.2007.11.005, 2008. 
[14] T. J. R. Hughes and A. N. Brooks. A multidimensional upwind scheme with no crosswind diffusion. In Finite element methods for convection dominated flows (Papers, Winter Ann. Meeting Amer. Soc. Mech. Engrs., New York, 1979), volume 34 of $A M D$, pages 19-35. Amer. Soc. Mech. Engrs. (ASME), New York, 1979.

[15] V. John and G. Matthies. MooNMD - a program package based on mapped finite element methods. Comput. Vis. Sci., 6(2-3):163-170, 2004.

[16] R. B. Kellogg and M. Stynes. Sharpened and corrected version of: Corner singularities and boundary layers in a simple convection-diffusion problem. J. Differential Equations, 213(1):81-120, 2005.

[17] R. B. Kellogg and M. Stynes. Sharpened bounds for corner singularities and boundary layers in a simple convection-diffusion problem. Appl. Math. Lett., 20(5):539-544, 2007.

[18] T. Linß and M. Stynes. Numerical methods on Shishkin meshes for linear convectiondiffusion problems. Comput. Methods Appl. Mech. Eng., 190(28):3527-3542, 2001.

[19] Linß, T. An upwind difference scheme on a novel Shishkin-type mesh for a linear convection-diffusion problem. J. Comput. Appl. Math., 110(1):93-104, 1999.

[20] Linß, T. Analysis of a Galerkin finite element method on a Bakhvalov-Shishkin mesh for a linear convection-diffusion problem. IMA J. Numer. Anal., 20(4):621-632, 2000.

[21] Linß, T. and Stynes, M. Asymptotic analysis and Shishkin-type decomposition for an elliptic convection-diffusion problem. J. Math. Anal. Appl., 261(2):604-632, 2001.

[22] G. Matthies. Local projection methods on layer-adapted meshes for higher order discretisations of convection-diffusion problems. Fakultät für Mathematik, RuhrUniversität Bochum, 2008.

[23] G. Matthies. Local projection stabilisation for higher order discretisations of convection-diffusion problems on Shishkin meshes. Adv. Comput. Math., DOI: 10.1007/s10444-008-9070-y, 2008.

[24] G. Matthies, P. Skrzypacz, and L. Tobiska. A unified convergence analysis for local projection stabilisations applied to the Oseen problem. M2AN Math. Model. Numer. Anal., 41(4):713-742, 2007.

[25] J. J. H. Miller, E. O'Riordan, and G. I. Shishkin. Fitted numerical methods for singular perturbation problems: Error estimates in the maximum norm for linear problems in one and two dimensions. World Scientific Publishing Co. Inc., River Edge, NJ, 1996.

[26] H.-G. Roos and T. Linß. Sufficient conditions for uniform convergence on layeradapted grids. Computing, 63(1):27-45, 1999. 
[27] M. Stynes and E. O'Riordan. A uniformly convergent Galerkin method on a Shishkin mesh for a convection-diffusion problem. J. Math. Anal. Appl., 214(1):36-54, 1997.

[28] M. Stynes and L. Tobiska. The SDFEM for a convection-diffusion problem with a boundary layer: optimal error analysis and enhancement of accuracy. SIAM J. Numer. Anal., 41(5):1620-1642, 2003. 